\title{
Über den Verlauf der Stickstoffoxydation bei elektrischen Entladungen in Gegenwart von Ozon
}

\author{
(II. Mitteilung) \\ von \\ Viktor Ehrlich und Franz Russ. \\ Aus dem Elektrochemischen Laboratorium der $\mathrm{k} . \mathrm{k}$. Staatsgewerbeschule \\ chemisch-technischer Richtung in Wien.
}

(Mit 7 Textfiguren.)

(Vorgelegt in der Sitzung am 9. Juli 1914.)

Einleitung. - MeBmethodik: a) Die Verwendung des Binantelektrometers von Dolezalek, b) Messungen im Stromkreis der Siemensröhre: 1. Messungen der elektrischen Größen im gesamten Sekundärkreis. 2. Feststellung der elektrischen Größien an den äußeren Belägen der Siemensröhre. 3. Messung der elektrischen Größen an der Gasstrecke. - Die Veränderungen der elektrischen Größen im Laufe der Entladung.

\section{Einleitung.}

Die elektrischen Entladungen liefern bei der Einwirkung auf Gemische von Stickstoff und Sauerstoff als Produkte Ozon und Stickoxyd, daneben auch Stickoxydul. Lichtbogen und Funken erzielen einen starken thermischen Effekt im Gase; hierbei kann Ozon infolge der großen Zerfallsgeschwindigkeit nur unter ganz besonderen Abkühlungsbedingungen vor vollständiger Zersetzung geschützt werden; Stickoxyd, für das die Bedingungen des Gleichgewichtes und des Zerfalles günstiger liegen, wird nach Abkühlung als $\mathrm{N}_{2} \mathrm{O}_{3}$, beziehungsweise als $\mathrm{NO}_{2} \rightleftarrows \mathrm{N}_{2} \mathrm{O}_{4}$ vorgefunden, und zwar in einer von den thermischen Bedingungen abhängigen Konzentration.

Chemie-Heft Nr. 5 . 
Wird aber der rein thermische Effekt der elektrischen Entladung vermieden und dies ist bei der Spitzen- und der sogenannten stillen elektrischen Entladung der Fall, so bleiben sowohl Ozon wie die gebildeten Stickstoffoxyde vor dem Zerfall bewahrt. Stickoxyd wird jedoch in Gegenwart von Ozon $z \mathrm{u}$ Stickstoffpentoxyd oxydiert, und zwar bei Zimmertemperatur mit außerordentlich großer Geschwindigkeit. Diese Tatsache hat nun, wie wir in einer früheren Arbeit ${ }^{1}$ zeigten, eine Koppelung der elektrischen und chemischen Wirkungen zur Folge. Eigenartige, hierdurch hervorgerufene Verhältnisse treten zutage, wenn man die Einwirkung der elektrischen Entladung auf ein in einen Ozonisator abgeschlossenes Stickstoff-Sauerstoffgemenge zeitlich verfolgt. Im Anbeginn der Entladung findet sich überwiegend Ozon neben geringen Mengen Stickstoffpentoxyd. Während aber die Ozonkonzentration einem Maximum zustrebt, das bald überschritten wird, steigt die $\mathrm{N}_{2} \mathrm{O}_{5}$-Menge unverändert an. In dem Zeitpunkt aber, in dem der Ozonwert auf Null gesunken ist, tritt ein jäher Abfall der Konzentration an Stickstoffpentoxyd ein; es wird über $\mathrm{N}_{2} \mathrm{O}_{4} \rightleftarrows \mathrm{NO}_{2}$ in Stickstoff und Sauerstoff zerlegt bis auf einen geringfügigen Wert; der schließlich auch bei andauernder Entladung unverändert erhalten bleibt.

In Ergänzung dieser rein chemischen Bestimmungen ermittelten wir nunmehr die physikalischen Größen und deren Veränderungen im Laufe der eben geschilderten Entladungsperioden, zumal eine systematische Verfolgung der elektrischen Bedingungen bei stetig geänderter Zusammensetzung des Gases in keinem uns bekannten Falle einer Entladungsreaktion vorlag.

\section{Meßmethodik.}

\section{a) Die Verwendung des Binantelektrometers von Dolezalek.}

Bei der Bestimmung der elektrischen Größen an Siemenslöhren handelt es sich um die Messung kleiner Ströme, die unter Hochspannung fließen, im allgemeinen einen sehr

1 Monatshefte für Chemie, 32, 917 (1911.) 
niedrigen Leistungsfaktor besitzen und deren Kurvenform schwer definierbar ist. Aus mehreren Gründen, vor allem aber, weil die Stromverhältnisse durch die Meßapparatur möglichst wenig geändert werden sollen, kommt nur die Anwendung von Elektrometern in Frage. Warburg und Leithäuser ${ }^{1}$ haben in ihren Arbeiten über Ozonisierung die Art der Schaltung des von ihnen verwendeten Quadrantelektrometers zwecks Messung von Stromstärke, Spannung und Gesamtleistung im Sekundärkreis dargelegt. Für unsere $Z$ wecke der steten Verfolgung dieser stark veränderlichen Größen schien die bei dem Quadrantelektrometer erforderliche Kommutation der Ladungen unbequem und wir entschlossen uns zur Benutzung des Binantelektrometers nach Dolezalek, ${ }^{2}$ das schon von Haber und $\mathrm{König}^{3}$ für energetische Messungen im Stromkreise des Hochspannungslichtbogens erprobt worden war. Außerdem ließ die für das Binantelektrometer angegebene Tatsache der Proportionạlität zwischen Ladung und Zeigerausschlag bei allen Schaltungen und unabhängig von der Kurvenform des Stromes dieses Instrument für unsere Zwecke besonders geeignet erscheinen. Schließlich fiel auch in die Wagschale, daß Zeigerinstrumente geliefert werden können, die einen besonderen stabilen Einbau nicht erfordern und deren Empfindlichkeit genügte.

Nun zeigte sich aber, daß bei den beiden uns gelieferten Instrumenten die Angaben über die Proportionalität zwischen Ladung und Ausschlag keineswegs zutrafen, daß vielmehr je nach der Ladung und je nach der Schaltung stark variierende Werte des Proportionalitätsfaktors erhalten wurden. Haber und König ${ }^{4}$ suchten diese Schwierigkeiten in einer Weise zu umgehen, welche aber die Unstimmigkeiten nicht behob. Es war somit vorerst nötig, durch eine langwierige Reihe von Eichungen die Bedingungen zu fixieren, welche die Anwendung des Instrumentes für unsere Zwecke gestatteten.

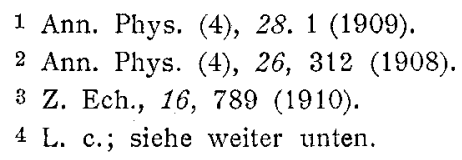




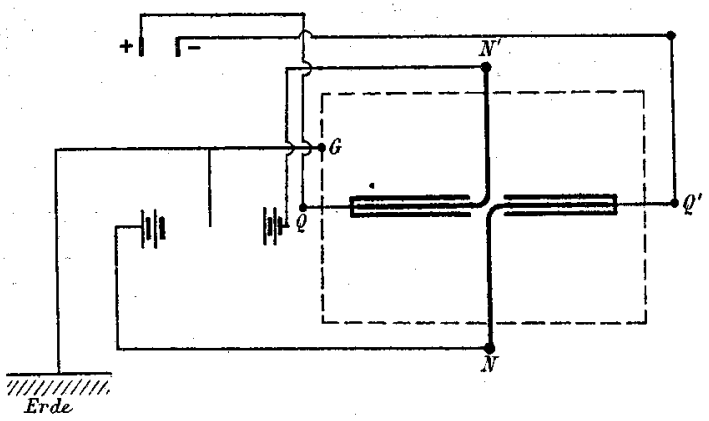

Schaltungsschema 1.

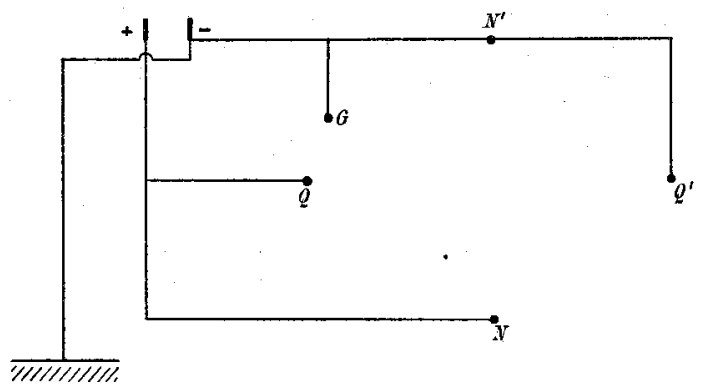

Schaltungsschema 2.

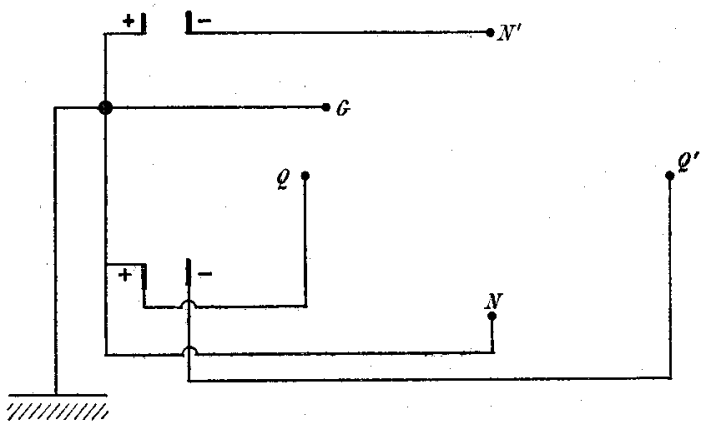

Schaltungsschema 3 .

Fig. 1.

$N N^{\prime}$ Nadel, $Q Q^{\prime}$ Schachtel, $G$ Gehäuse.

Die Eichungen erfolgten teils unter Zuhilfenahme einer Krüger'schen Meßbatterie von 100 Elementen, teils mit Hilfe von Gleichspannungen bis 220 Volt durch steten Vergleich mit 
einem Westonpräzisionsinstrument, teils mit 50 periodischem Wechselstrom bis 66 Volt. Die Nullage des Instrumentes wurde vor jeder Meßreihe nach Erdung sämtlicher Teile ermittelt. ${ }^{1}$

1. Wird an die Nadel eine symmetrisch geteilte Hilfsladung $N$ gelegt, die in der Mitte geerdet ist (vgl. Fig. 1, Schaltungsschema 1), so ergibt sich für jede Schachtelladung $Q$ Proportionalität innerhalb der Meßfehler. Der auf die Nullage reduzierte Ausschlag $a$ ist somit:

$$
a=p \cdot N \cdot Q \text {. }
$$

Der Proportionalitätsfaktor $p$ ändert sich mit der Nadelladung:

Tabelle I.

\begin{tabular}{|c|c|c|c|c|c|}
\hline$N$ & $Q$ & $\begin{array}{c}a \\
\text { gemessen }\end{array}$ & $\stackrel{a}{\text { reduziert }}$ & $p$ & $\underset{\text { Mittel }}{p}$ \\
\hline 101 & $\begin{array}{c}0 \\
6 \cdot 04 \\
12 \cdot 1 \\
26 \cdot 6 \\
37 \cdot 0 \\
48 \cdot 0 \\
62 \cdot 7\end{array}$ & $\begin{array}{r}0 \cdot 2 \\
8 \cdot 6 \\
17 \cdot 2 \\
36 \cdot 8 \\
51 \cdot 5 \\
66 \cdot 5 \\
85 \cdot 4\end{array}$ & $\begin{array}{l}0 \\
8 \cdot 4 \\
17 \cdot 0 \\
36 \cdot 6 \\
51 \cdot 3 \\
66 \cdot 3 \\
85 \cdot 2\end{array}$ & $\begin{array}{r}-\overline{01375} \\
1385 \\
1365 \\
1375 \\
1365 \\
1345\end{array}$ & $\begin{array}{r}0.0133 \\
\pm 1 \%\end{array}$ \\
\hline $60 \cdot 6$ & $\begin{array}{c}0 \\
12 \cdot 17 \\
26 \cdot 7 \\
39 \cdot 3 \\
62 \cdot 6 \\
83 \cdot 0 \\
98 \cdot 6\end{array}$ & $\begin{array}{r}0 \cdot 4 \\
10 \cdot 4 \\
22 \cdot 2 \\
32 \cdot 4 \\
52 \cdot 6 \\
68 \cdot 6 \\
79 \cdot 4\end{array}$ & $\begin{array}{c}0 \\
10 \cdot 0 \\
21 \cdot 8 \\
32 \cdot 0 \\
52 \cdot 2 \\
68 \cdot 2 \\
79 \cdot 0\end{array}$ & $\begin{array}{r}- \\
0 \cdot 01355 \\
-1350 \\
1345 \\
1375 \\
1355 \\
1325\end{array}$ & $\begin{array}{l}0.0135 \\
\pm 2 \%\end{array}$ \\
\hline $20 \cdot 2$ & $\begin{array}{c}0 \\
26 \cdot 6 \\
42 \cdot 2 \\
63 \cdot 4 \\
84 \cdot 0 \\
98 \cdot 2 \\
116 \cdot 5\end{array}$ & $\begin{array}{r}0 \cdot 3 \\
7 \cdot 5 \\
11 \cdot 6 \\
17 \cdot 0 \\
22 \cdot 3 \\
26 \cdot 3 \\
31 \cdot 4\end{array}$ & $\begin{array}{c}0 \\
7 \cdot 2 \\
11 \cdot 3 \\
16 \cdot 7 \\
22 \cdot 0 \\
26 \cdot 0 \\
31 \cdot 1\end{array}$ & $\begin{array}{r}-\overline{0134} \\
133 \\
130 \\
130 \\
131 \\
132\end{array}$ & $\begin{array}{l}0.0132 \\
\pm 1 \%\end{array}$ \\
\hline
\end{tabular}

1 Die Zuleitungsdrähte waren mit geerdeten Hüllen aus Stanniolpapier umwickelt. Diese Vorsichtsmaßregel erwies sich jedoch im allgemeinen als unnötig. 
2. Für idiostatische Schaltung, bei der die Spannung einerseits an die Nadel, andrerseits an die Schachtel gelegt wird und der eine Pol geerdet ist (vgl. Fig, 1, Schaltungsschema 2), gilt die Beziehung:

$$
N=Q ; \quad a=p \cdot Q^{2} .
$$

Es ergaben sich Abweichungen von der Proportionalität.

Tabelle 2.

\begin{tabular}{|c|c|c|c|c|}
\hline$N=Q$ & $\stackrel{a}{\text { gemessen }}$ & $\stackrel{a}{a}$ reduziert & $p$ & Mittel $p$ \\
\hline \multicolumn{5}{|c|}{ 1. Spannungsquelle: Mebbatterie } \\
\hline $\begin{array}{c}0 \\
10 \cdot 1 \\
20 \cdot 2 \\
30 \cdot 3 \\
40 \cdot 4 \\
50 \cdot 5 \\
60 \cdot 6 \\
70 \cdot 7 \\
80 \cdot 8\end{array}$ & $\begin{array}{r}0 \cdot 6 \\
0 \cdot 7 \\
4 \cdot 9 \\
11 \cdot 8 \\
21 \cdot 7 \\
34 \cdot 7 \\
50 \cdot 5 \\
69 \cdot 4 \\
89 \cdot 3\end{array}$ & $\begin{array}{l}0 \\
1 \cdot 3 \\
5 \cdot 5 \\
12 \cdot 4 \\
22 \cdot 3 \\
35 \cdot 3 \\
51 \cdot 1 \\
70 \cdot 0 \\
89 \cdot 9\end{array}$ & $\begin{array}{c}-\overline{0128}) \\
135 \\
135 \\
137 \\
138 \\
139 \\
140 \\
138\end{array}$ & $\begin{array}{l}0.0136 \\
\pm 2 \%\end{array}$ \\
\hline \multicolumn{5}{|c|}{ 2. Spannungsquelle: Maschinengleichstrom } \\
\hline $\begin{array}{l}0 \\
12 \cdot 08 \\
26 \cdot 7 \\
34 \cdot 7 \\
44 \cdot 3 \\
56 \cdot 7 \\
62 \cdot 7 \\
73 \cdot 4 \\
75 \cdot 6\end{array}$ & $\begin{array}{r}0 \cdot 4 \\
2 \cdot 4 \\
10 \cdot 0 \\
16 \cdot 6 \\
26 \cdot 7 \\
44 \cdot 4 \\
54 \cdot 7 \\
73 \cdot 5 \\
78 \cdot 2\end{array}$ & $\begin{array}{l}0 \\
2 \cdot 0 \\
9 \cdot 6 \\
16 \cdot 2 \\
26 \cdot 3 \\
44 \cdot 0 \\
54 \cdot 3 \\
73 \cdot 1 \\
77 \cdot 8\end{array}$ & $\begin{array}{c}-\overline{-} \\
(0.0137) \\
135 \\
135 \\
134 \\
137 \\
138 \\
136 \\
136\end{array}$ & $\begin{array}{r}0.0136 \\
\pm 1.5 \%\end{array}$ \\
\hline \multicolumn{5}{|c|}{ 3. Spannungsquelle: Maschinenwechselstrom } \\
\hline $\begin{array}{c}0 \\
15 \cdot 0 \\
24 \cdot 0 \\
34 \cdot 2 \\
43 \cdot 6 \\
55 \cdot 2 \\
65 \cdot 5 \\
76 \cdot 3\end{array}$ & $\begin{array}{r}+0.6 \\
3.8 \\
8 \cdot 4 \\
16 \cdot 4 \\
26 \cdot 4 \\
41 \cdot 2 \\
58 \cdot 5 \\
79.6\end{array}$ & $\begin{array}{l}0 \\
3 \cdot 2 \\
7 \cdot 8 \\
15 \cdot 8 \\
25 \cdot 8 \\
40 \cdot 6 \\
57 \cdot 9 \\
79 \cdot 0\end{array}$ & $\begin{array}{c}-\overline{0142} \\
(0 . \\
135 \\
135 \\
136 \\
134 \\
135 \\
136\end{array}$ & $\begin{array}{l}0.0136 \\
\pm 1 \%\end{array}$ \\
\hline
\end{tabular}


Die Beziehung wurde graphisch in der Weise festgelegt, daß $p$ in Abhängigkeit vom Nadelausschlag dargestellt wurde. Es bestand ein kleiner, aber bestimmter Unterschied, je nachdem das eine oder das andere Hälftenpaar an Erde gelegt wurde.

3. Bei der Leistungsmessung (vgl. Fig. 1, Schaltungsschema 3) werden zwei verschiedene Spannungen an Nadel und Schachtel gelegt, von denen die eine der Gesamtspannung, die andere der Stromstärke proportional ist. Das Elektrometer gibt hierbei wie jedes Wattmeter einen Ausschlag, der dem Produkte der Momentanwerte entspricht und den Leistungsverbrauch unabhängig von der Kurvenform des Wechselstromes darstellt:

$$
a=p \cdot Q \cdot N=p^{\prime} . L .
$$

Je ein Pol der beiden Ladungen wurden vereinigt und geerdet. Nach den Angaben von Dolezalek ist das Instrument unabhängig von der Kurvenform des Stromes zu verwenden und gibt bei Wechselstrom die Effektivwerte an.

Für Wechselstrommessung kommt natürlich Schaltung 1 nicht in Frage. Schaltung 2 (idiostatische Schaltung) ist

1. für Messungen von Spannungen, bei Verwendung eines geeigneten Spannungsteilers auch zur Messung von hohen Spannungen $z \mathrm{u}$ verwenden.

2. Sie dient zur Bestimmung der Stromstärke durch Messung der Spannung an den Enden eines bekannten induktionsfreien Widerstandes auf Grund des Ohm'schen Gesetzes: Besondere Versuchsreihen mit Wechselstrom zeigten durch Vergleich mit elektromagnetischen Instrumenten, daß die für die Gleichspannung gewonnenen Faktoren tatsächlich ohne weiteres für Wechselstrom Geltung besitzen (vgl. Tabelle 2).

Für Leistungsmessungen dient Schaltung 3. Haber und König ${ }^{1}$ haben für die jeweiligen Ladungen an der Nadel, beziehungsweise Schachtel, die Faktoren bei idiostatischer Schaltung festgestellt und diese Faktoren auch für die Berechnung der Leistung herangezogen. Demgegenüber zeigten aber unsere Eichungen, daß die Faktoren bei idiostatischer 
Schaltung für die Leistungsschaltung nicht ohne weiteres verwendet werden dürfen, daß man vielmehr bei einigermaßen erheblichen Phasenverschiebungen zu beträchtlich verschiedenen Werten gelangt, je nachdem man die an der Nadel oder die an der Schachtel liegende Spannung der Berechnung des Faktors zugrunde legt. Auch $\mathrm{Haber}$ und König führen $\mathrm{Ab}$ weichungen an, die aber nicht sehr bedeutend waren, weil die Phasenverschiebungen beim Lichtbogen viel geringer sind als im Stromkreis der Siemensröhre.

Wir mußten daher besondere Eichungen vornehmen. Hierbei wurden die Nadelausschläge verzeichnet, die sich ergaben, wenn bei konstanter Nadelladung die Schachtelladung variiert wurde, beziehungsweise bei konstanter Schachtelladung die Nadelladung. Die Berechnung zeigte, daß der Faktor $p$ sehr veränderlich ist; im allgemeinen ist er für jede Schachtelund jede Nadelladung verschieden. Es wurden Eichkurven aufgenommen, die für konstante Nadel- oder für konstante Schachtelladung den Faktor $p$ in Abhängigkeit vom Nadelausschlag darstellen. Arbeitet man mit Gleichstrom oder mit Wechselstrom, bei dem Strom und Spannung in Phase laufen, so läßt sich aus dem Nadelausschlag und einer der beiden Ladungen der zugehörige Faktor aus den Eichkurven ohne weiteres ableiten. Liegt aber Wechselstrom mit Phasenverschiebung vor, so gehört zu der bekannten Nadel(Schachtel) ladung ein anderer Wert der Schachtel(Nadel) ladung, als den Eichkurven entspricht. Daraus entspringt eine Unsicherheit, die um so größer wird, je größer die Phasenverschiebung ist. Diese Unsicherheit mußte möglichst beseitigt werden.

Nun hat die Höhenlage der Nadel in der Schachtel einen Einfluß auf die Größe und die Veränderlichkeit der Faktoren $p$. Die Höhenlage der Nadel ist zwar durch eine Marke am Nadelstiel kenntlich gemacht, doch ist diese Einstellung viel zu grob; schon minimale Verschiebungen haben einen starken Einfluß. Durch Versuchsmessungen gelingt es aber stets, eine Höhenlage ausfindig $z u$ machen, bei der die Variation der Faktoren $p$ innerhalb gewisser Grenzen der Skala am geringsten wird. So konnte z. B. in einem Falle bei Veränderung der Nadel- und Schachtelladungen von 60 bis 
100 Volt und zwischen den Skalenteilstrichen 35 und 75 (die Skala ist in 90 Teile geteilt) ein innerhalb der Meßfehler ausreichend konstanter Faktor erzielt werden (vgl. Fig. 2). Innerhalb dieses Bereiches war dann die Unsicherheit behoben. Messungen jenseits dieser Grenzen waren nach Tunlichkeit $z \mathrm{u}$ vermeiden; war dies unmöglich, so wurde das geometrische Mittel aus den beiden Faktoren genommen, die für den betreffenden Ausschlag $z u$ den Effektivspannungen an Nadel und an Schachtel gehörten.

Der Vorgang der Eichung des Instrumentes gestaltete sich nun folgendermaßen: Durch vergleichende Messungen wurde zuerst jene Höhenlage der Nadel ausfindig gemacht, bei der die geringsten Veränderungen der Faktoren $p$ auftraten. Sodann wurde eine Reihe von Messungen unter Veränderung der Nade1-, sowie der Schachtelspannungen von 10 bis 100 Volt in Abständen von $10 \mathrm{zu} 10 \mathrm{Volt}$ vorgenommen. Die Ergebnisse wurden in der Weise graphisch festgelegt, daß die Faktoren $p$ einerseits bei konstanter Nadelladung, andrerseits bei konstanter Schachtelladung in Abhängigkeit vom Nadelausschlag graphisch dargestellt wurden (Fig. 2).

Derartige Eichungen mußten aber bei Inanspruchnahme des Instrumentes wiederholt werden. ${ }^{1}$ Veränderungen bei längerer Benutzung ergeben sich infolge Verlängerung des Aufhängedrahtes durch Dehnung.

Die Beschränkungen zur Erzielung sicherer Meßresultate bestehen in der Vermeidung kleiner Nadelausschläge; ferner sind Nadelladungen außerhalb des Bereiches von 50 bis 150 Volt, Schachtelladungen von 20 bis 120 Volt unbedingt auszuschließen.

Hieraus ergeben sich Schwierigkeiten bei sehr kleinem Leistungsfaktor, weil es dann auch bei den höchsten zulässigen Ladungen nicht mehr gelingt, die untere Grenze des Nadelausschlages $z u$ erreichen. Um trotzdem derartige Messungen $\mathrm{zu}$ ermöglichen, haben wir gegebenenfalls den Leistungsfaktor des gesamten Stromkreises künstlich erhöht, indem ein bekannter selbstinduktionsfreier Widerstand in den

1 Vgl. Dolezalek, 1. c.; Haber und König, l. c. 


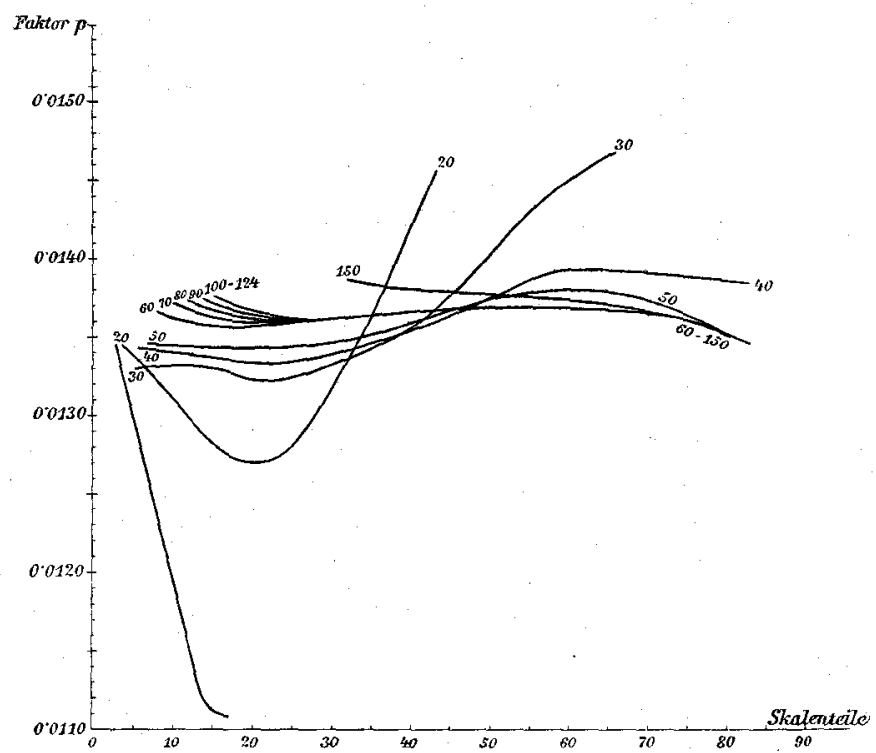

a) Nadelladung konstant.

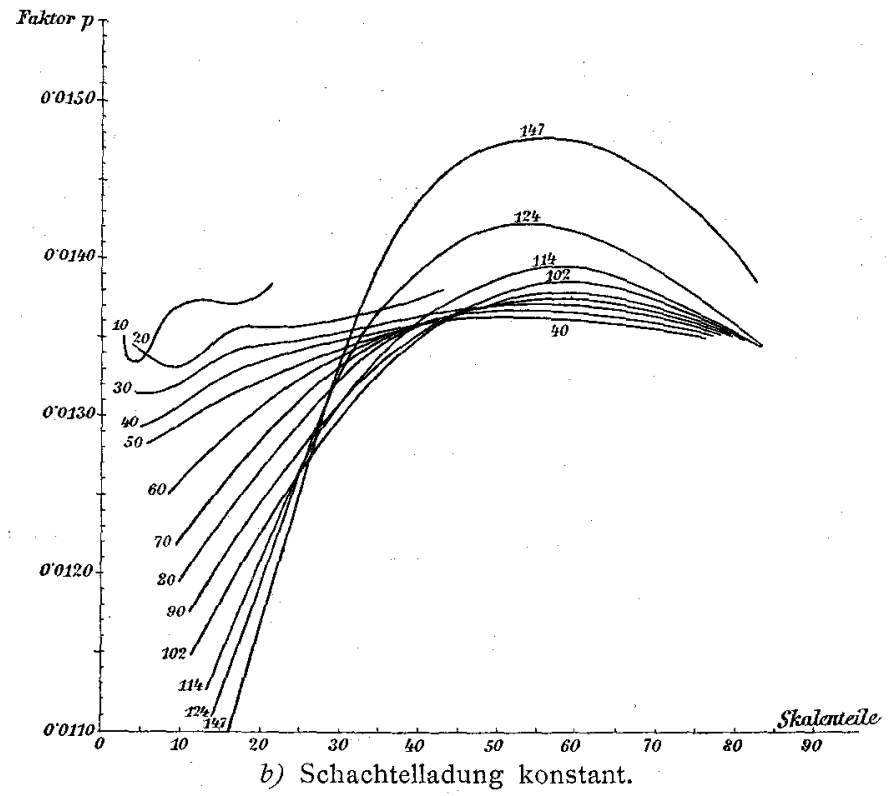

Fig. 2.

Eichkurven für die Leistungsmessung.

Die den einzelnen. Kurven beigesetzten Zahlen bedeuten die bei der Eichung konstant gebaltene Nadel-, beziehungsweise Schachtelladung in Volt. 
Stromkreis eingeschaltet und die auf den Widerstand entfallende Teilleistung in Abzug gebracht wurde. Dies hat es uns ermöglicht, selbst Leistungsfaktoren der Größe 0.2 mit hinreichender Genauigkeit zu bestimmen.

\section{b) Messungen im Stromkreise der Siemensröhre. \\ 1. Messungen der elektrischen Größen des gesamten Sekundärkreises.}

Die Spannungsmessung erfolgte teils mit Hilfe von Hochspannungsvoltmetern der Firma Hartmann \& Braun (bis 10.000 Volt) oder durch Messung der Primärspannung nach Bestimmung des jeweiligen Transformationsverhältnisses des Transformators, teils auf elektrometrischem Wege. Die elektrometrische Messung geschah in der Weise, daß die Sekundärklemmen des Transformators durch einen großen induktionsfreien Widerstand geschlossen und an einem aliquoten Teile der Spannungsabfall festgestellt wurde.

Als Spannungsteiler diente ein Flüssigkeitswiderstand (Borsäure-Mannitlösung mit Platinelektroden), der aus mehreren hintereinander geschalteten Systemen U-förmig gebogener Glastöhren von $0.6 \mathrm{~cm}$ lichter Weite gebildet wurde. Durch Einhängen der Röhren in Isolatoröl konnte eine störende Erwärmung auch bei hoher Belastung vermieden werden. Der Gesamtwiderstand betrug entweder $2.10^{6}$ oder $4.10^{6} \mathrm{Ohm}$; bei dem ersten Rohrsystem waren an einem Ende mehrere Platindrähte mit herausragenden Ösen in kleinen Abständen eingeschmolzen; die Endelektrode wurde mit dem geerdeten Pol des Transformators verbunden; an den einzelnen Platindrähten konnten verschiedene Teilspannungen abgenommen werden. Das Teilungsverhältnis wurde vor und nach jeder Versuchsreihe bestimmt.

Die Schaltung des Elektrometers war die idiostatische; ist das Spannungsverhältnis am Spannungsteiler $n$, der Nadelausschlag $a$, der Faktor des Instrumentes für diesen Nadelausschlag $p$, so ist die Gesamtspannung

$$
E=n \cdot \sqrt{\frac{a}{p}} .
$$


Zur Strommessung wurde in den Stromkreis der Siemensröhre ein bekannter induktionsfreier Widerstand $\left(W_{1}\right)$ gelegt. Als Meßwiderstände dienten induktionsfrei gewickelte Metallwiderstände der Firma Hartmann \& Braun von 1000 bis $100.000 \mathrm{Ohm}$, zuweilen auch ein Flüssigkeitswiderstand. Das Elektrometer ergab bei idiostatischer Schaltung durch Messung der Spannung an den Enden dieses Widerstandes die Stromstärke

$$
J=\frac{1}{W_{1}} \sqrt{\frac{a}{p}}
$$

Zur Leistungsmessung muß eine der Stromstärke proportionale und phasengleiche Spannung, z. B. an die Nadel,

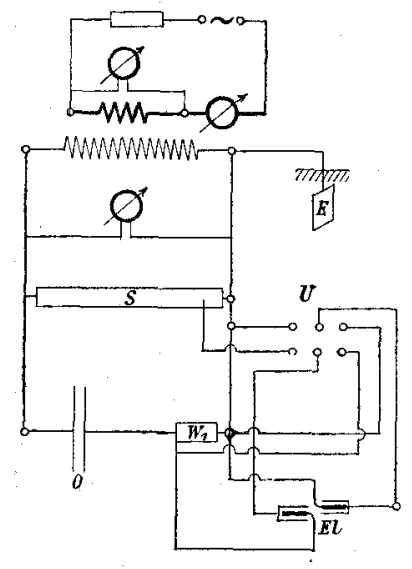

Fig. 3.

Schaltungsschema.

$S$ Spannungsteiler.

O Ozonröhre.

El Elektrometer.

$U$ Umschalter.

$E$ Erde. eine der Gesamtspannung proportionale und phasengleiche Teilspannung an die Schachtel gelegt - werden oder umgekehrt. $\mathrm{Zu}$ diesem $Z$ wecke wurde die Nadel an die Enden des wie bei der Strommessung geschalteten Widerstandes $W_{1}$ gelegt, die Schachtelhälften mit den Zwischenkontakten des Spannungsteilers verbunden. Je eine Hälfte der Nadel und der Schachtel war geerdet.

Das Schaltungsschema ergibt sich aus der nebenstehenden Fig. 3. Diese Schaltung ermöglicht unter Verwendung des Umschalters $U$ mit demselben Instrumente in kurzer Aufeinanderfolge Leistung und Stromstärke zu messen. Dies war nach den Ergebnissen der Eichungen deswegen erforderlich, weil für die Leistungsmessung unter Umständen die an der Nadel und an der Schachtel liegenden Spannungen bekannt sein mußten.

In der einen Lage des Umschalters sind sowohl Nadel wie Schachtel an die Enden des Widerstandes $W_{1}$ geschaltet; 
der Ausschlag gibt somit die Stromstärke. In der anderen Lage bleibt (z. B.) die Schachtel an den Klemmen des Widerstandes $W_{1}$, der leicht $z u$ variieren ist, während die Nadel an den Spannungsteiler geschaltet wird; der Ausschlag ist dann der Leistung proportional. Da die Gesamtspannung sowie das Teilungsverhältnis $n$ jeweils bekannt waren und die Stromstärke vor und nach jeder Leistungsmessung ermittelt wurde, da somit die an Nadel und Schachtel liegenden Spannungen gegeben waren, ließ sich auf Grund der Eichungen aus dem Nadelausschlag die Leistung berechnen.

\section{Feststellung der elektrischen Größen an den äußeren Belägen der Siemensröhre.}

Die in der dargestellten Weise ausgeführten Messungen ergeben auf direktem Wege den Strom $(J)$, die Leistung $(L)$ und die Spannung $(E)$ im Gesamtkreis. Ein Teil dieser Leistung und Spannung entfällt aber auf den aus meßtechnischen Gründen der Siemensröhre vorgeschalteten Normalwiderstand $W_{1}$. Die im Ozonisator verbrauchte Leistung $\left(L_{a}\right)$ ergibt sich aus der Gesamtleistung nach Abzug des Betrages $J^{2} . W_{1}$. Zur Berechnung der Spannung an der Siemensröhre muß berücksichtigt werden, daß die Teilspannung an dem selbstinduktionsfreien Widerstand $W_{1}$ mit dem Strom in Phase läuft, während Strom und Spannung am Ozonisator im allgemeinen sehr stark gegeneinander verschoben sind. Die Gesamtspannung, deren rechnerische Phasenverschiebung gegen den Strom aus der Leistungsmessung bekannt ist, setzt sich geometrisch aus den beiden Teilspannungen zusammen. Die vektorielle $\mathrm{Zu}$ sammenstellung der Gesamtspannung und der Teilspannung am Widerstand führt $z u$ der folgenden bequemen Näherungsformel zur Berechnung der Spannung an der Siemensröhre:

$$
E_{a}=E-\frac{L \cdot W_{1}}{E}
$$

3. Messungen der elektrischen Größen an der Gasstrecke.

Die elektrischen Größen an den äußeren Belägen der Siemensröhren sind für die unter Einwirkung der elektrischen 
Entladungen stattfindenden Gasreaktionen nur indirekt von Bedeutung; wichtig sind hingegen die Verhältnisse an der Gasstrecke selbst. Wir knüpften an die Darlegungen von Warburg und Leithäuser ${ }^{1}$ über die Wirkungsweise der Siemensröhre als der eines zusammengesetzten Kondensators an und konnten sodann den Weg festlegen, der es gestattet, aus den Messungen an den äußeren Belägen jeweils auch die Werte der elektrischen Größen an der Gasstrecke zu berechnen. Wir haben hierüber an anderer Stelle berichtet.? Unsere Siemensröhren wurden von zwei konzentrischen Glasröhren gebildet, welche die Gasstrecke einschlossen. Man kann sich die Siemensröhre schematisch als ein System vorstellen, in dem ein reines Dielektrikum (Glas) und die Gasstrecke hintereinander geschaltet sind. Letztere wirkt unterhalb der sogenannten Durchschlagsspannung ebenfalls vornehmlich als Dielektrikum, oberhalb der Durchschlagsspannung zum größten Teil als Leiter, dies um so mehr, je größer die Ionisierung ist. Um nun das Glasdielektrikum in dem verwendeten Siemensrohr gesondert zu definieren, wurde die Gasstrecke unter Ersatz durch einen guten Leiter (Quecksilber, verdünnte Schwefelsäure) ausgeschaltet und dann einerseits das Verhältnis von Strom zu Spannung (Kapazität der Glasröhren), andrerseits die durch das Glas hervorgerufene Phasenverschiebung zwischen Strom und Spannung (Leistungsfaktor) festgestellt. Der gesamte Strom, der die Siemensröhre, also auch die Gasstrecke durchfließt, ist gleichzeitig Lade-, beziehungsweise Entladestrom des vorgeschalteten Glaskondensators; somit ergibt sich mit Hilfe der vorher bestimmten Kapazität des Glases aus der Stromstärke sofort auch die Spannung am Glase. Die Gesamtspannung $\left(E_{a}\right)$ des Systems setzt sich geometrisch aus der Teilspannung am Glasdielektrikum $\left(E_{D}\right)$ und an der Gasstrecke $\left(E_{i}\right)$ zusammen; nach Messung der Gesamtspannung und des Gesamtleistungsfaktors $\frac{L_{a}}{E_{a} . J}$ sind durch vektorielle

1 E. Warburg, Verh. D. phys. G., 1903, 382 ; E. Warburg und G. Leithäuser, Ann. Phys. (4), 28, 1 (1909).

2 Z. Ech., 19, 330 (1913). 
Zusammensetzung dieser Werte mit den zugehörigen Größen am Dielektrikum schließlich die Spannung $\left(E_{i}\right)$, der Leistungsfaktor $\left(k_{i}\right)$ und somit auch die Leistung an der Gasstrecke $\left(L_{i}=J . E_{i} k_{i}\right)$ gegeben. Die vektorielle Berechnung läßt sich entweder graphisch nach Fig. 4 oder mit ausreichender Genauigkeit nach der Näherungsformel

$$
E_{i}^{2}=E_{a}^{2}+E_{D}^{2}-2 E_{a} \cdot E_{D}\left(\sqrt{1-k_{a}^{2}}+k_{a} \cdot k_{D}\right)
$$

ausführen.

An allen verwendeten Siemensröhren haben wir die charakteristischen Kurven für den Verlauf von Stromstärke,

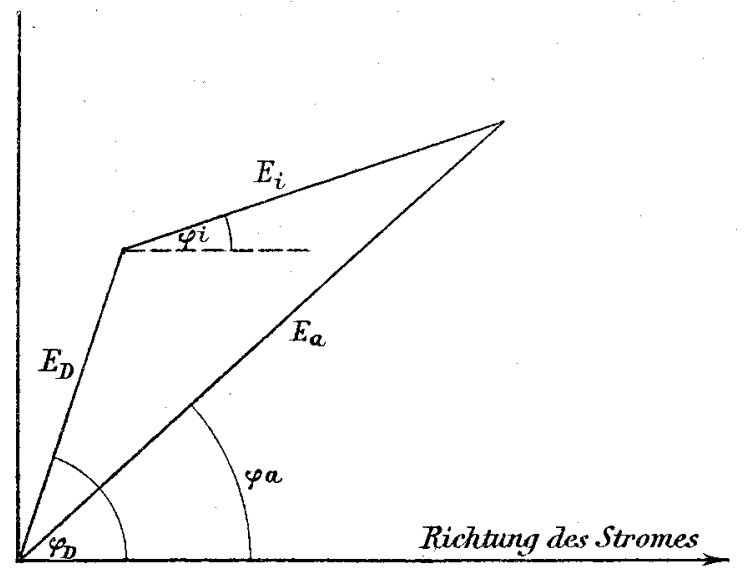

Fig. 4.

Leistung und Leistungsfaktor bei wachsender äußerer Spannung aufgenommen und aus den an den äußeren Belägen gewonnenen Werten die charakteristischen Kurven für die Gasstrecke berechnet. Hierbei mußte berücksichtigt werden, daß schon geringfügige Änderungen in der chemischen $\mathrm{Zu}$ sammensetzung des Gases beträchtliche Abweichungen verursachen. Die charakteristische Kurve wurde daher bei so stark strömendem Gase (Luft) aufgenommen, daß innerhalb der Zeitdauer der Messung eine merkliche Verschiebung der Verhältnisse nicht eintrat. Zur Veranschaulichung des Verlaufes der Charakteristiken entnehmen wir einer früheren Arbeit ${ }^{1}$ das umstehende Kurvenblatt (Fig. 5), das für ein be-

1 Ehrlich und Rulss, Z. Ech,, 19, 335 (1913). 


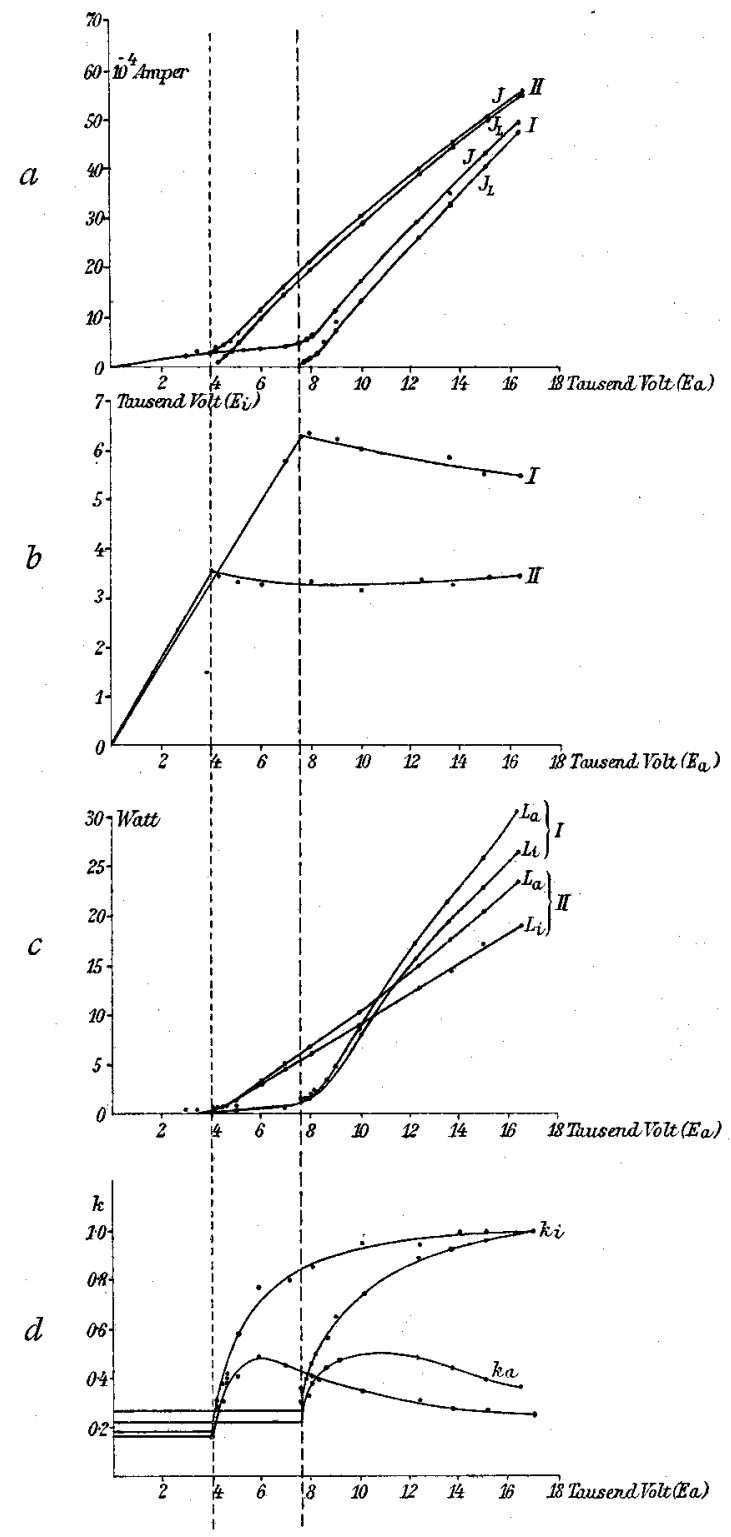

Fig. 5.

a) Gesamtstrom - Ionisierungsstrom - Gesamtspannung.

b) Spannung an der Gasstrecke - Gesamtspannung.

c) Gesamtleistung-Leistung an der Gasstrecke-Gesamtspannung.

d) Leistungsfaktoren - Gesamtspannung. 
stimmtes Siemensrohr die Änderungen der elektrischen Größen bei 1 Atmosphäre Gasdruck (I), beziehungsweise 1/2 Atmosphäre (II) wiedergibt.

Schon geringfügige Beimengung von fremden Gasen beeinflussen die Durchschlagsspannung, d. i. jene geringste Spannung, bei der unter den gegebenen Bedingungen die Entladung eben einsetzt. Sie ist durch den plötzlichen starken Anstieg des Leistungsverbrauches wie der Stromstärke gekennzeichnet. Der Verlauf der Spannung an der Gasstrecke, die unterhalb der Durchschlagsspannung der Stromstärke proportional bleibt, weist beim Durchschlagspotential einen Knickpunkt auf und zeigt dann einen um so schärferen $\mathrm{Ab}$ fall, je höher die Durchschlagsspannung liegt; bei niedrigen Werten ist ein schwacher Anstieg bemerkbar.

Besondere Aufmerksamkeit verdient die Variation der Leistungsfaktoren des Gesamtsystems und der Gasstrecke. Beide sind unterhalb der kritischen Spannung konstant. Der Leistungsfaktor an der Gasstrecke steigt von dem niedrigen Werte unterhalb der kritischen Spannung (hervorgerufen durch Verschiebungsströme und geringfügige Leitungsströme) nach Einsetzen der Entladung steil an. Er näbert sich in allen Fällen rasch dem Werte 1. Die starke Vergrößerung des Leistungsfaktors am Gase nach Eintritt der Entladung bewirkt zunächst auch eine Erhöhung des Gesamtleistungsfaktors; dieser erreicht bei wachsender äußerer Spannung ein Maximum, nach dessen Überschreitung infolge des relativen Zurücktretens der Spannung an der Gasstrecke ein Abfall gegen jenen Grenzwert stattfindet, der dem Glase unter Kurzschließung der Gasstrecke zukommt. ${ }^{1}$

Für die weiteren Betrachtungen ist der Ionisierungsstrom von besonderer Wichtigkeit. Das Gas wirkt auch nach Eintritt der Entladung noch als Dielektrikum, zumal in jeder Periode des Wechselstromes Ionisierung immer nur nach Überschreiten der jeweiligen Durchschlagsspannung ein- und nach Unterschreitung einer Minimalspannung immer wieder

1 Vg1. Z. Ech., 1. c., 331. 
aussetzt. Bei großer Gesamtstromstärke tritt der dielektrische Verschiebungsstrom relativ stark zurück und wird durch die gewöhnlich eintretende Erniedrigung der Spannung am Gase auch absolut verringert. Sonst kann der Verschiebungsstrom auch Werte erreichen, die gegenüber dem Ionisierungsstrom in Betracht zu ziehen sind. Für die chemischen Reaktionen kommt aber nur der ionisierende Anteil des. Stromes in Betracht. Einer einwandfreien messenden Festlegung der Teilströme stellen sich Schwierigkeiten in den Weg. Die nicht ionisierenden Teilströme bestehen in Leitungsströmen unterhalb der Durchschlagsspannung, die der vorliegenden Ionisierung entsprechen, und in Verschiebungsströmen. Somit ließe sich die Größe der Ströme und die zugehörige Phasenverschiebung nur für den jeweilig herrschenden Grad der Ionisierung im Gase definieren, der nicht ohne weiteres fixiert werden kann. Wenn aber das Gas den Entladungsraum mit sehr großer Geschwindigkeit durchströmt, so daß angenommen werden kann, daß der Entladung immer nur Frischgas ausgesetzt wird, läßt sich ein Anhaltspunkt für den nicht ionisierenden Anteil des Gesamtstromes aus den Messungen unterhalb der Durchschlagsspannung gewinnen. Wir haben in diesem Sinne für die Berechnung der Größe des Verschiebungsstromes die Kapazität der Gasstrecke $\frac{J}{E_{i}}$ unterhalb der kritischen Spannung und ferner die Verschiebung dieses Stromes gegen die Gasspannung, d.i. den zugehörigen konstanten Leitungsfaktor $\left(k_{v}\right)$ zugrunde gelegt. Die vektorielle Zusammenstellung dieser Werte mit den jeweils bestimmten Größen des Gesamtstromes liefert den Leitungsstrom nach der folgenden Formel, die ohneVernachlässigung einzelner Glieder gebraucht werden muß:

$$
J_{L}=\sqrt{J^{2}+J_{v}^{2}-2 J . J_{v}\left(\sqrt{l-k_{i}^{2}-\bar{k}_{v}^{2}+k_{i}^{2} k_{v}^{2}}+k_{i} \cdot k_{v}\right)}
$$

Die auf den Ionisierungsstrom entfallende Teilleistung berechnet sich $\mathrm{zu}$

$$
L_{L}=L_{i}-E_{i} . J_{v} \cdot k_{v} .
$$

Die Berechnung des Leistungsfaktors des Ionisierungsstromes in Bezug auf die zugehörige Teilleistung stößt auf 
die Schwierigkeit, daß auch nur ein Teil der Gasspannung ionisierend wirkt. Die Messungen weisen darauf hin, daß Leitungsstrom und erzeugende Teilspannung in Phase laufen.

Die Berechnung des Ionisierungsstromes für die Entladung im ruhenden oder schwachströmenden Gase läßt sich nach den obigen Gesichtspunkten nicht vornehmen, weil für diesen Fall die Werte der Verschiebungsströme ohne neue Annahme nicht bekannt sind. Nun konnten wir eine große Anzahl von Messungen unter den verschiedensten Bedingungen ausführen und berechnen; hierbei ergab sich empirisch, daß der Ausdruck

$\frac{L_{i}}{E_{i} . J_{L}}\left(\frac{\text { Gesamtleistung an der Gasstrecke }}{\text { Gesamtspannung an der Gasstrecke.Ionisierungsstrom }}\right)$ mit ausreichender Genauigkeit stets den Wert 1 besitzt. Da der wahre Leistungsfaktor nach

$$
\frac{L_{L}}{E_{L} \cdot J_{L}}\left(\frac{\text { Ionisierende Teilleistung }}{\text { Ionisierende Teilspannung. Ionisierungsstrom }}\right)
$$

zu berechnen wäre und ebenfalls den Wert 1 besitzt, so erkennt man beim Vergleich der beiden Ausdrücke, daß das Verhältnis $\frac{L_{L}}{L_{i}}$ mit dem Verhältnis $\frac{E_{L}}{E_{i}}$ ziemlich übereinstimmt.

Tabelle 3.

Stark strömende Luft.

\begin{tabular}{|c|c|c|c|c|c|c|c|}
\hline 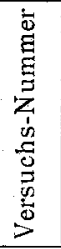 & 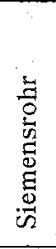 & 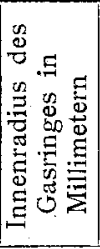 & 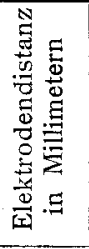 & 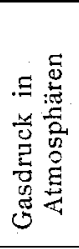 & $\begin{array}{c}\text { Meßbereich } \\
\text { für } J_{L} \text { in } \\
10-4 \text { Ampere }\end{array}$ & $\begin{array}{c}\text { Mittel- } \\
\text { wert } \\
\text { von } \\
\frac{L_{i}}{E_{i} . J_{L}}\end{array}$ & $\begin{array}{c}\text { Mittlere Ab- } \\
\text { weichungen, } \\
\text { berechnet } \\
\text { nach der } \\
\text { Methode } \\
\text { der kleinsten } \\
\text { Quadrate }\end{array}$ \\
\hline 22 & Il & $17 \cdot 5$ & $4 \cdot 0$ & 1 & $5 \cdot 0-47: 9$ & $1 \cdot 00$ & \pm 0.02 \\
\hline 23 & II & $17 \cdot 5$ & $4 \cdot 0$ & $0 \cdot 5$ & $2 \cdot 4-55 \cdot 7$ & 0.94 & \pm 0.02 \\
\hline 67 & IV & $17 \cdot 75$ & $1 \cdot 15$ & 1 & $5 \cdot 7-51 \cdot 9$ & $(0 \cdot 98)$ & \pm 0.01 \\
\hline 80 & $\mathrm{~V}$ & $17 \cdot 5$ & $12 \cdot 5$ & 0.5 & $16 \cdot 7-48 \cdot 2$ & $1 \cdot 00$ & \pm 0.01 \\
\hline 126 & VI & $6 \cdot 3$ & $6 \cdot 3$ & 1 & $16 \cdot 5-58 \cdot 5$ & $(0.96)$ & \pm 0.04 \\
\hline
\end{tabular}


Somit läßt sich der Leitungsstrom $J_{L}$ in erster Annäherung einfach berechnen, wenn die Gesamtleistung an der Gasstrecke durch die Gesamtspannung an der Gasstrecke dividiert wird: $J_{L}=\frac{L_{i}}{E_{i}}$.

Die elektrischen Größen, vor allem die Größen an der Gasstrecke lassen sich in der beschriebenen Weise feststellen, urd zwar werden

A. direkt gemessen:

1. vor und nach der Versuchsgruppe: die Konstanten des Dielektrikums (Leistungsfaktor $k_{D}$ und Kapazität $\frac{E_{D}}{J}$ );

2. während der Entladung: Gesamtspannung $E_{a}$, Strom $J$ und Gesamtleistung $L_{a}$ (Leistungsfaktor $k_{a}$ );

$B$. indirekt berechnet:

1. Teilspannung am Gase $E_{i}$, Leistungsverbrauch im Gase $L_{i}$, Leistungsfaktor $k_{i}$;

2. Ionisierungsstrom $J_{L}$.

Die Veränderung der elektrisehen Größen im Laufe der Entladung.

In unserer ersten Arbeit ${ }^{1}$ haben wir den Verlauf der Ozon- und Stickstoffpentoxydbildung im abgeschlossenen Gasgemisch an Hand der Druckänderungen und der chemischen Messungen näher verfolgt. Wir bezeichneten den ersten $\mathrm{Ab}$ schnitt der Entladungsvorgänge bis zum Eintritte des Druckminimums als Ozonperiode, weil hier in Gegenwart von überschüssigem Ozon eine stete Nachbildung von Stickoxyd stattfindet; den zweiten Abschnitt, bei dem die Zersetzung des $\mathrm{N}_{2} \mathrm{O}_{5}$ über $\mathrm{N}_{2} \mathrm{O}_{4} \rightleftarrows \mathrm{NO}_{3}$ in Stickstoff und Sauerstoff erfolgt, nannten wir kurzerhand die $\mathrm{NO}_{2}$-Periode.

Die folgenden Versuche wurden in der gleichen Anordnung ausgeführt, wie sie p. 927 unserer ersten Arbeit

1 Monatshefte für Chemie, 32, 917 (1911). 
abgebildet ist. Auch die Arbeitsweise, die rechnerische Verwertung der Druckmessungen, sowie die analytische Methodik blieben unverändert. Wir arbeiteten zunächst mit sinusförmigem Wechselstrom von 50 Perioden; der in einen Induktionsapparat auf Hochspannung transformiert wurde. Die elektrischen Messungen erfolgten in gewissen Zeitabständen abwechselnd mit den Druckablesungen am Quecksilbermanometer. Nur in einem Punkte ist eine Änderung der Arbeitsweise $z a$ besprechen: Wir führten früher die Entladungen in der Weise aus, daß der im Primärkreis dem Transformator vorgeschaltete Regulierwiderstand während des ganzen Versuches konstant gehalten wurde; dann war stets bis zum Druckminimum ein geringer Anstieg der Sekundärspannung, bei Wechselstrom auch ein Anstieg der Stromstärke im Primärkreis $z u$ verzeichnen. ${ }^{1}$ Zur Vereinfachung der elektrischen Messungen wurde bei den folgenden Versuchen die Sekundärspannung konstant gehalten. Hierbei ergab sich eine kleine Verschiebung, die berücksichtigt werden mußte. Wir hatten nämlich bei unserer früheren Arbeitsweise gefunden, daß der $\mathrm{Zu}$ wachs der Stickoxydkonzentration während der Ozonperiode bis $z \mathrm{ul}$ erheblichen $\mathrm{N}_{2} \mathrm{O}_{5}$-Konzentrationen konstant blieb; dadurch war eine sehr einfache Berechnung der Konzentration an Ozon und Stickstoffpentoxyd aus den Druckmessungen gegeben, da im Druckminimum neben $\mathrm{N}_{2}$ und $\mathrm{O}_{2}$ praktisch nur $\mathrm{N}_{2} \mathrm{O}_{5}$, vorher nur $\mathrm{O}_{3}$ und $\mathrm{N}_{2} \mathrm{O}_{5}$ angenommen werden konnte. Das Konstanthalten der Spannung während eines Entladungsversuches bedeutete nun bei den höheren $\mathrm{N}_{2} \mathrm{O}_{5}$ Konzentrationen eine kleine Erniedrigung der Spannung gegenüber den Bedingungen der früheren Versuche, die sich in einer Verringerung der Stickoxydbildungsgeschwindigkeit äußern mußte. Daher waren jedenfalls noch besondere chemische Messungen vorzunehmen und dies geschah in folgender Weise: Ein Gesamtversuch wurde unter bestimmter sekundärer Spannung in seinem zeitlichen Verlaufe verfolgt, wobei die Druckablesungen und elektrischen Messungen festgelegt wurden. Unmittelbar darauf wurden der Reihe nach mehrere Versuche

1 L. c., p. 745. 
unter gleichen Bedingungen ausgeführt, aber innerhalb der Ozonperiode nach wechselnder Einwirkungsdauer der Entladung abgebrochen; die Reaktionsprodukte wurden sodann analytisch bestimmt. Den Abschluß der Versuchsreihe bildete dann nochmals ein Gesamtversuch. ${ }^{1}$ Die aus den Zwischenversuchen gewonnenen analytischen Ergebnisse wurden nun auf den einen der beiden Gesamtversuche unter Zugrundelegung der zugehörigen korrigierten und reduzierten Druckablesungen bezogen.

Wir haben die Veränderung der elektrischen Größen in Beziehung $\mathrm{zu}$ den. chemischen Vorgängen in demselben Siemensrohr (II) untersucht, das wir auch für unsere rein chemischen Messungen zumeist verwendet hatten. Wir geben nochmals die Dimensionen: ${ }^{2}$

Länge des Entladungsraumes $30.0 \mathrm{~cm}$

$$
\left.\begin{array}{l}
r_{1}=16 \cdot 4 \mathrm{~mm} \\
r_{2}=17 \cdot 5 \mathrm{~mm}
\end{array}\right\} \text { inneres Glasrohr }
$$

Elektrodendistanz....... $4.0 \mathrm{~mm}$ Gasstrecke

$$
\left.\begin{array}{l}
r_{3}=21.5 \mathrm{~mm} \\
r_{4}=22.8 \mathrm{~mm}
\end{array}\right\} \text { äuBeres Glasrohr }
$$

Elektrodenoberfläche des

$$
\text { inneren Glasrohres .... } 330.0 \mathrm{~cm}^{2}
$$

Abgeschlossenes Gasvolumen

$$
\left(0^{\circ}, 760 \mathrm{~mm}\right) \ldots \ldots \ldots . \quad 141 \mathrm{~cm}^{3}
$$

Die für dieses Siemensrohr zur Zeit der Durchführung der folgenden Versuche charakteristischen elektrischen Größen, die für rasch strömende, $\mathrm{P}_{2} \mathrm{O}_{5}$-trockene Luft aufgenommen wurden, sind bereits in Fig. 5 graphisch dargestellt. ${ }^{3}$

Vor der folgenden Versuchsreihe wurden die Konstanten des Glasdielektrikums bestimmt. Es ergab sich die Kapazität

$$
\frac{J}{E} \text { zu } 3 \cdot 54( \pm 0 \cdot 01) \cdot 10^{-7}\left(\frac{\text { Ampere }}{\text { Volt }}\right)
$$

1 Dieser letzte Versuch zeigte stets gegenüber dem ersten Versuch eine kleine. Verscbiebung in den zeitlichen Bedingungen.

2 L. c., p. 715.

3 Die zugrunde liegenden Zahlenwerte finden sich: Z. Ech., 19, 334 (1913). 
demgegenüber zeigte der Leistungsfaktor $k_{D}$ in diesem Siemensrohr bei niedrigen Stromstärken einen ausgeprägten Gang, während sich in anderen untersuchten Fällen auch für diesen Wert über den gesamten Meßbereich konstante Zahlen ergaben:

\begin{tabular}{rl}
\multicolumn{1}{c}{$J$} & \multicolumn{1}{c}{${ }^{k_{D}}$} \\
$7.10^{-4}$ & 0.065 \\
$18 . »$ & 0.09 \\
$35 . »$ & 0.055 \\
ab $40 . »$ & $0.048 \pm 0.001$ konstant.
\end{tabular}

Wir geben nun die für eine bestimmte Sekundärspannung durchgeführte vollständige Versuchsreihe wieder. Die Versuche 41 und 47 sind die Gesamtversuche, die den Anfang, beziehungsweise den Schluß der Reihe bildeten. Die Ergebnisse der chemischen Konzentrationsbestimmungen am Ende der einzelnen $Z$ wischenversuche sind auf den Versuch 47 bezogen, den wir mit allen Details des Versuchsprotokolls zugleich als Beispiel für Messungen und Berechnungen wiedergeben. Zum Vergleich wird auch der zu Anfang der Versuchsreihe ausgeführte Versuch 41 in gekürzter Form angeführt. Die $Z$ wischenversuche sind nur in Form einer zusammenfassenden Tabelle verzeichnet.

In den folgenden Tabellen bedeuten:

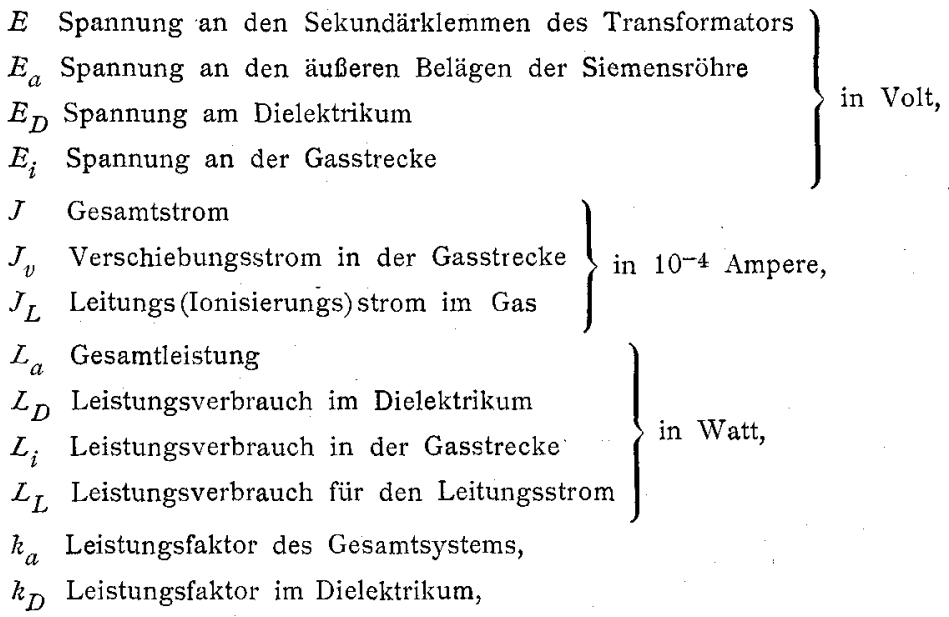


$k_{i}$ Leistungsfaktor in der Gasstrecke,

$k_{v}$ Leistungsfaktor in der Gasstrecke unterhalb des Minimumpotentials,

$k_{L}$ Leistungsfaktor für den Leitungsstrom.

\section{Ferner gelten:}

$W_{1}$ in den Stromkreis der Siemensröhre geschalteter Normalwiderstand in $104 \mathrm{Ohm}$,

$a_{J}$ Elektrometerausschiag (auf Null reduziert) bei der Strommessung,

$p_{J}$ der zu $a_{J}$ gehörige Faktor laut Eichkurve,

$a_{L}$ Elektrometerausschlag (reduziert) bei der Leistungsmessung,

$p_{L}$ der $z \mathfrak{u} a_{L}$ gehörige Faktor laut Eichkurve,

$n$ Spannungsverbältnis am Spannungsteiler,

$L$ vom Elektrometer angezeigte Gesamtleistung. 
Verlauf der Stickstoffoxydation.

Tabelle 4.

Versuch 47.

$E=12250$ Volt $E_{a}=12200$ Volt.

Luft, Gasdruck $733 \cdot 1 \mathrm{~mm} \mathrm{Hg}$.

a) Druckablesungen.

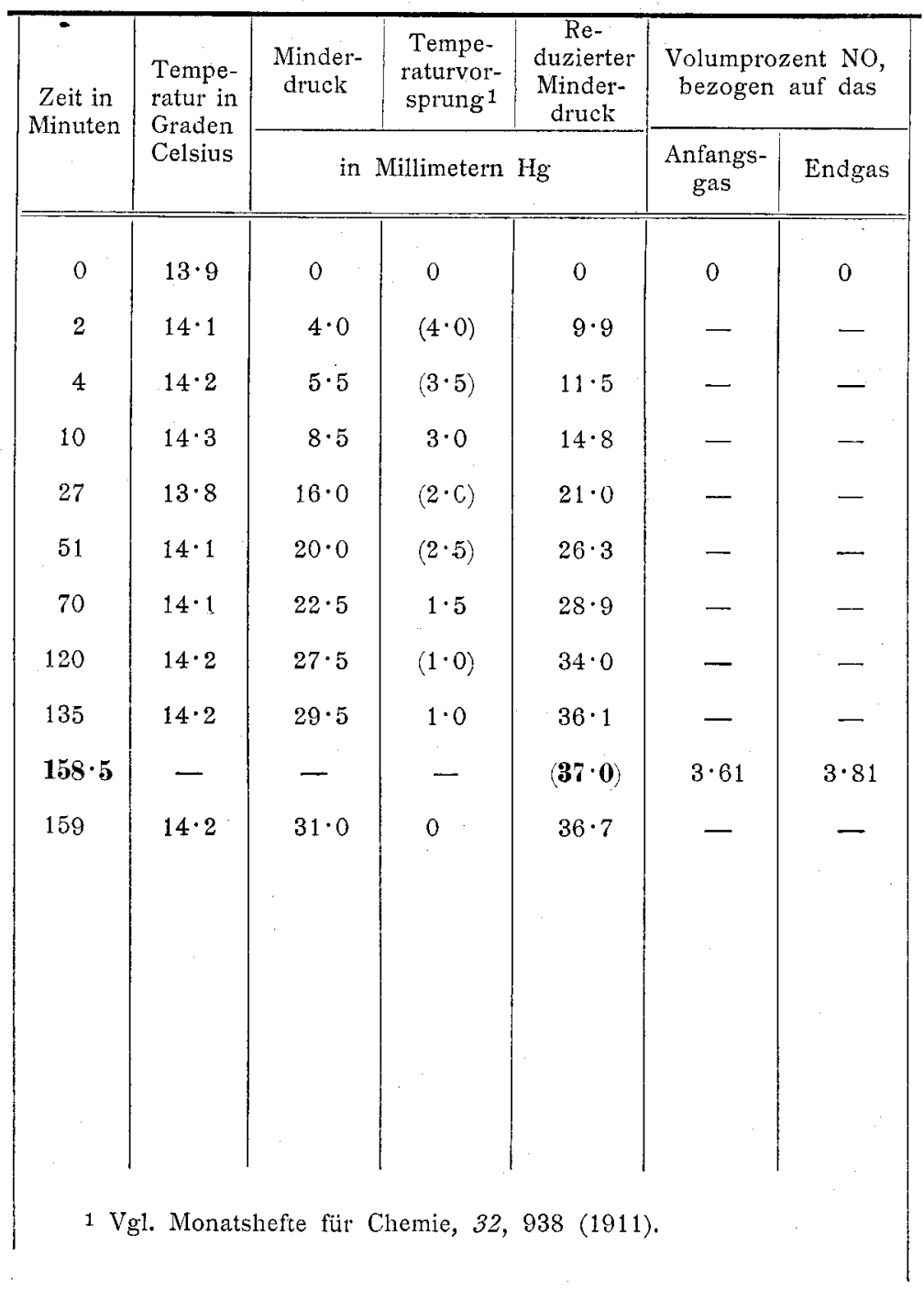


b) Elektrische

\begin{tabular}{|c|c|c|c|c|c|c|c|}
\hline Zeit & $a_{J}$ & $p_{J}$ & $J$ & ${ }^{a}{ }_{L}$ & $p_{L}$ & $n$ & $L$ \\
\hline 0 & - & - & $(27: 0)$ & - & - & - & - \\
\hline 1 & $57 \cdot 2$ & $0 \cdot 01360$ & $21 \cdot 6$ & - & - & - & - \\
\hline $1 \cdot 5$ & - & - & $(20 \cdot 8)$ & $37 \cdot 2$ & $0 \cdot 0136$ & 141 & $12 \cdot 86$ \\
\hline 3 & $45 \cdot 2$ & 0.01350 & $19 \cdot 3$ & 一 & - & - & - \\
\hline 5 & $72 \cdot 2$ & 0.01350 & $18 \cdot 2$ & - & - & - & - \\
\hline 6 & - & - & $(17 \cdot 9)$ & $44 \cdot 2$ & 0.0136 & 141 & $11 \cdot 45$ \\
\hline 8 & $62 \cdot 2$ & 0.01360 & $16 \cdot 9$ & - & - & - & - \\
\hline $9 \cdot 5$ & - & - & $(16 \cdot 8)$ & $39 \cdot 6$ & 0.0136 & 141 & $10 \cdot 27$ \\
\hline $12 \cdot 5$ & - & - & $(15 \cdot 9)$ & $38 \cdot 7$ & 0.0136 & 141 & $10 \cdot 04$ \\
\hline 14 & $53 \cdot 2$ & 0.01360 & $15 \cdot 6$ & $\longrightarrow$ & - & - & - \\
\hline $16 \cdot 5$ & $51 \cdot 2$ & 0.01360 & $15 \cdot 3$ & - & - & - & - \\
\hline 17 & - & - & $(15 \cdot 2)$ & $36 \cdot 2$ & 0.0136 & 141 & $9 \cdot 38$ \\
\hline 22 & - & - & $(14 \cdot 6)$ & $35 \cdot 2$ & 0.0136 & 141 & $9 \cdot 12$ \\
\hline 25 & $43 \cdot 7$ & 0.01350 & $14 \cdot 2$ & - & - & 一 & - \\
\hline 29 & $41 \cdot 9$ & 0.01350 & $14 \cdot 0$ & - & - & - & - \\
\hline 30. & - & - & - & $33 \cdot 2$ & 0.01345 & $141 \cdot 5$ & $8 \cdot 73$ \\
\hline 40 & $一$ & - & - & $30 \cdot 2$ & $0 \cdot 01345$ & 142 & $7 \cdot 96$ \\
\hline 41 & $36 \cdot 2$ & $0 \cdot 01340$ & $13 \cdot 0$ & - & - & - & - \\
\hline 52 & $33 \cdot 2$ & 0.01330 & $12 \cdot 5$ & - & - & - & - \\
\hline 53 & - & - & - & $27 \cdot 2$ & 0.01345 & 142 & $7 \cdot 18$ \\
\hline 68 & - & - & $(11 \cdot 9)$ & $25 \cdot 8$ & 0.01345 & 143 & $6 \cdot 87$ \\
\hline 70 & $29 \cdot 0$ & 0.01320 & $11 \cdot 8$ & - & - & 一 & - \\
\hline 118 & $24 \cdot 8$ & 0.01310 & $10 \cdot 9$ & - & - & - & - \\
\hline 119 & - & - & - & $21 \cdot 6$ & 0.0134 & 144 & $5 \cdot 80$ \\
\hline 134 & - & - & - & $20 \cdot 8$ & $0 \cdot 0134$ & 145 & $5 \cdot 62$ \\
\hline 135 & $24 \cdot 4$ & 0.01310 & $10 \cdot 8$ & - & - & - & -- \\
\hline 142 & $23 \cdot 2$ & 0.01305 & $10 \cdot 6$ & - & - & - & 一 \\
\hline 146 & - & - & $(10 \cdot 5)$ & $20 \cdot 0$ & 0.0134 & 145 & $5 \cdot 42$ \\
\hline 156 & $21 \cdot 8$ & 0.01300 & $10 \cdot 3$ & - & - & - & - \\
\hline 158 & - & - & $(10 \cdot 2)$ & $19 \cdot 8$ & 0.0134 & 145 & $5 \cdot 36$ \\
\hline $158 \cdot 5$ & $26 \cdot 2$ & 0.01310 & $11 \cdot 2$ & - & - & - & - \\
\hline
\end{tabular}


Messungen.

\begin{tabular}{|c|c|c|c|c|c|c|c|c|}
\hline$J^{2} W_{1}$ & $L_{a}$ & $k_{a}$ & $E_{D}$ & $E_{i}$ & $L_{i}$ & $k_{i}$ & $J_{L}$ & Zeit \\
\hline- & $(15 \cdot 5)$ & $0 \cdot 47$ & 7650 & 6200 & $14 \cdot 1$ & $0 \cdot 84$ & $22 \cdot 8$ & 0 \\
\hline- & - & - & - & - & - & - & - & 1 \\
\hline $0 \cdot 17$ & $12 \cdot 7$ & 0.505 & 5880 & 7450 & $11 \cdot 6$ & $0 \cdot 76$ & $15 \cdot 6$ & $1 \cdot 5$ \\
\hline- & - & - & - & - & - & - & - & 3 \\
\hline- & - & 一 & - & - & - & - & - & 5 \\
\hline $0 \cdot 13$ & $11 \cdot 32$ & 0.52 & 5050 & 8150 & $10 \cdot 4$ & 0.71 & $12 \cdot 8$ & 6 \\
\hline - & - & -- & - & - & - & - & 一 & 8 \\
\hline 0.11 & $10 \cdot 16$ & 0.50 & 4750 & 8300 & $9 \cdot 5$ & 0.69 & $11 \cdot 5$ & $9 \cdot 5$ \\
\hline $0 \cdot 10$ & $9 \cdot 94$ & 0.515 & 4500 & 8500 & $9 \cdot 2$ & 0.68 & $10 \cdot 8$ & $12 \cdot 5$ \\
\hline- & - & - & - & - & - & - & - & 14 \\
\hline - & - & - & - & - & - & - & - & $16 \cdot 5$ \\
\hline 0.09 & $9 \cdot 29$ & 0.505 & 4300 & 8600 & $8 \cdot 7$ & 0.67 & $10 \cdot 1$ & 17 \\
\hline 0.09 & $9 \cdot 03$ & 0.51 & 4150 & 8750 & $8: 4$ & 0.66 & $9 \cdot 6$ & 22 \\
\hline- & - & - & - & - & - & - & - & 25 \\
\hline$\cdots$ & 一 & - & - & - & - & - & - & 29 \\
\hline 0.08 & $8 \cdot 65$ & 0.51 & 4000 & 8850 & $8 \cdot 2$ & 0.66 & $9 \cdot 3$ & 30 \\
\hline 0.08 & $7 \cdot 89$ & 0.495 & 3700 & 9050 & $7 \cdot 4$ & 0.63 & $8 \cdot 2$ & 40 \\
\hline- & - & - & - & - & - & - & - & 41 \\
\hline- & - & - & - & - & - & - & - & 52 \\
\hline 0.06 & $7 \cdot 12$ & 0.47 & 3350 & 9150 & $6 \cdot 6$ & $0 \cdot 58$ & $7 \cdot 2$ & 53 \\
\hline 0.06 & $6 \cdot 81$ & 0.47 & 3550 & 9300 & $6 \cdot 4$ & $0 \cdot 54$ & $6 \cdot 9$ & 68 \\
\hline- & - & - & - & - & - & - & 一 & 70 \\
\hline- & - & - & - & - & - & - & - & 118 \\
\hline 0.05 & $5 \cdot 75$ & $0 \cdot 435$ & 3100 & 9400 & $5 \cdot 4$ & 0.53 & $5 \cdot 7$ & 119 \\
\hline 0.05 & $5 \cdot 57$ & $0 \cdot 425$ & 3050 & 9450 & $5 \cdot 3$ & 0.52 & $5 \cdot 6$ & 134 \\
\hline- & - & - & - & - & - & - & - & 135 \\
\hline- & - & - & 一 & - & - & - & - & 142 \\
\hline $0 \cdot 04$ & $5 \cdot 38$ & 0.42 & 3000 & 9470 & 5.2 & $0 \cdot 52$ & $5 \cdot 5$ & 146 \\
\hline- & - & - & - & - & - & - & - & 156 \\
\hline 0.04 & $5 \cdot 32$ & 0.42 & 2900 & 9600 & $5 \cdot 1$ & $0 \cdot 52$ & $5 \cdot 3$ & 158 \\
\hline- & - & - & - & - & - & - & - & $158 \cdot 5$ \\
\hline
\end{tabular}


Tabelle 5.

Versuch 41.

$E_{a}=12200$ Volt.

Luft, Gasdruck $746 \mathrm{~mm} \mathrm{Hg}$ :

\begin{tabular}{|c|c|c|c|c|c|c|c|c|}
\hline $\begin{array}{l}\text { Zeit in } \\
\text { Minuten }\end{array}$ & $\begin{array}{l}\text { Tempe- } \\
\text { ratur in } \\
\text { Graden } \\
\text { Celsius }\end{array}$ & $\mid \begin{array}{c}\text { Redu- } \\
\text { zierter } \\
\text { Min- } \\
\text { der- } \\
\text { druck }\end{array}$ & $J$ & $L_{a}$ & $k_{a}$ & $E_{i}$ & $L_{i}$ & $k_{i}$ \\
\hline 0 & $15 \cdot 7$ & 0 & $(28)$ & $(16 \cdot 5)$ & 0.48 & 6150 & $15 \cdot 0$ & 0.89 \\
\hline 3 & - & - & $19 \cdot 5$ & - & - & - & - & - \\
\hline 5 & $16 \cdot 1$ & $11 \cdot 1$ & $(18 \cdot 5)$ & $12 \cdot 4$ & 0.55 & 8150 & $11 \cdot 5$ & $0 \cdot 76$ \\
\hline 8 & - & - & $17 \cdot 1$ & - & - & - & - & - \\
\hline 9 & - & 一 & $(16 \cdot 8)$ & $11 \cdot 3$ & $0 \cdot 56$ & 8540 & $10 \cdot 6$ & 0.75 \\
\hline 15 & $15 \cdot 7$ & $16 \cdot 6$ & $(15 \cdot 2)$ & $10 \cdot 4$ & $0 \cdot 56$ & 8800 & $9 \cdot 8$ & $0 \cdot 73$ \\
\hline 16 & - & - & $15 \cdot 1$ & - & - & 一 & - & - \\
\hline 25 & $15 \cdot 5$ & $20 \cdot 2$ & $13 \cdot 9$ & $8 \cdot 17$ & $0 \cdot 49$ & 8900 & $7 \cdot 7$ & $0 \cdot 62$ \\
\hline 35 & - & - & $13 \cdot 0$ & $7 \cdot 55$ & 0.47 & 9000 & $7 \cdot 0$ & $0 \cdot 60$ \\
\hline 47 & $15 \cdot 3$ & $25 \cdot 1$ & $12 \cdot 3$ & $6 \cdot 91$ & $0 \cdot 46$ & 9200 & $6 \cdot 4$ & 0.57 \\
\hline 62 & - & - & $11 \cdot 4$ & $6 \cdot 42$ & 0.46 & 9400 & $6 \cdot 0$ & $0 \cdot 56$ \\
\hline 80 & $15 \cdot 3$ & $30 \cdot 4$ & $11 \cdot 3$ & $6 \cdot 06$ & 0.44 & 9420 & $5 \cdot 6$ & 0.53 \\
\hline 104 & - & - & $10 \cdot 7$ & $5 \cdot 61$ & $0 \cdot 43$ & 9500 & $5 \cdot 3$ & $0 \cdot 52$ \\
\hline 105 & $15 \cdot 4$ & $32 \cdot 3$ & - & - & - & - & - & - \\
\hline 130 & $15 \cdot 6$ & $34 \cdot 0$ & $10 \cdot 3$ & $5 \cdot 31$ & 0.425 & 9600 & $5 \cdot 0$ & 0.51 \\
\hline 145 & $15 \cdot 7$ & $35 \cdot 4$ & - & - & - & - & - & - \\
\hline 146 & - & - & $10 \cdot 1$ & $5 \cdot 21$ & 0.42 & 9650 & $5 \cdot 0$ & 0.51 \\
\hline 164 & - & $(36 \cdot 5)$ & $(9 \cdot 8)$ & $5 \cdot 04$ & $0 \cdot 42$ & 9700 & $4 \cdot 8$ & $0 \cdot 505$ \\
\hline 165 & - & - & $11 \cdot 0$ & - & - & - & - & - \\
\hline 166 & $15 \cdot 9$ & $29 \cdot 5$ & $(13 \cdot 5)$ & $7 \cdot 33$ & $0 \cdot 455$ & 8900 & $6 \cdot 8$ & 0.57 \\
\hline 170 & $16 \cdot 2$ & $13 \cdot 1$ & $(21 \cdot 0)$ & $12 \cdot 46$ & $0 \cdot 49$ & 7400 & $11 \cdot 4$ & $0 \cdot 73$ \\
\hline 171 & - & - & $(22)$ & $13 \cdot 6$ & 0.50 & 7200 & $12 \cdot 5$ & $0 \cdot 78$ \\
\hline 172 & - & - & $(22 \cdot 7)$ & - & - & - & - & - \\
\hline 173 & - & - & $(23 \cdot 8)$ & $14 \cdot 0$ & 0.49 & 6850 & $12 \cdot 8$ & 0.79 \\
\hline 174 & - & - & $24 \cdot 3$ & - & 一. & - & - & - \\
\hline 175 & $16 \cdot 8$ & $7 \cdot 9$ & $(25)$ & $14 \cdot 4$ & 0.47 & 6500 & $13 \cdot 1$ & 0.80 \\
\hline
\end{tabular}


Tabelle 6.

Versuche 41 bis 47 .

$E_{a}=12200$ volt.

\begin{tabular}{|c|c|c|c|c|}
\hline $\begin{array}{c}\text { Versuchs- } \\
\text { Nummer }\end{array}$ & $\begin{array}{c}\text { Dauer der } \\
\text { Entladung } \\
\text { in Minuten }\end{array}$ & $\begin{array}{c}\text { Reduzierter } \\
\text { Minderdruck } \\
\text { in Millimetern } \\
\mathrm{Hg}\end{array}$ & $\begin{array}{c}\text { Volum- } \\
\text { prozent } \mathrm{O}_{3}\end{array}$ & $\begin{array}{c}\text { Volum- } \\
\text { prozent No }\end{array}$ \\
\hline & $\begin{array}{c}\text { bezogen auf das Anfangs- } \\
\text { gas }\end{array}$ \\
\hline 45 & 4 & $12 \cdot 1$ & $2 \cdot 35$ & $0 \cdot 34$ \\
44 & 5 & $13 \cdot 2$ & $2 \cdot 53$ & $0 \cdot 38$ \\
43 & 10 & $14 \cdot 6$ & $2 \cdot 48$ & $0 \cdot 55$ \\
46 & 30 & $22 \cdot 6$ & $(2 \cdot 27)$ & - \\
48 & 30 & $22 \cdot 1$ & $(2 \cdot 3)$ & $0 \cdot 89$ \\
42 & 60 & $27 \cdot 9$ & $(1 \cdot 4)$ & $1 \cdot 61$ \\
47 & 159 & $36 \cdot 7$ & 0 & $3 \cdot 61$ \\
41 & 164 & $36 \cdot 5$ & - & - \\
& & & & \\
\hline
\end{tabular}

Im Zusammenhang mit den einzelnen in verschiedenen Stadien der Entladungsvorgänge abgebrochenen Versuchen wurde jeweils vor der analytischen Konzentrationsbestimmung der Wert der Durchschlagsspannung einige Minuten nach Abstellung der Entladung festgestellt; bei langsam gesteigerter äußerer Spannung ist der Eintritt der Entladung am Auftreten des charakteristischen Geräusches scharf zu erkennen.

Das Verhältnis zwischen Gesamtspannung $\left(E_{a}\right)$ und Spannung an der Gasstrecke $\left(E_{i}\right)$ bleibt bis zum Eintritte der Entladung konstant und variiert auch innerhalb einer längeren Versuchsreihe nur wenig. Die Kenntnis dieses Quotienten gestattet, die Spannung an der Gasstrecke (unterhalb der Durchschlagsspannung) aus der Gesamtspannung zu berechnen. Diese bei Aufnahme der charakteristischen Größen festgestellte Zahl betrug z. B. während dieser Versuchsreihe $0 \cdot 85 \pm 1 \%$.

Wir geben in der folgenden Zusammenstellung die Veränderung der Durchschlagsspannung im Laufe der Entladungsvorgänge: 
Tabelle 7 .

\begin{tabular}{|c|c|c|c|c|c|c|c|}
\hline \multirow{3}{*}{ 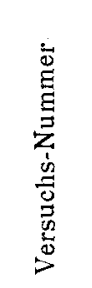 } & \multirow{3}{*}{ 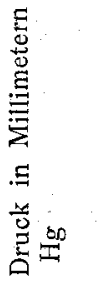 } & \multirow{3}{*}{ 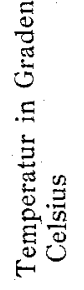 } & \multirow{3}{*}{ 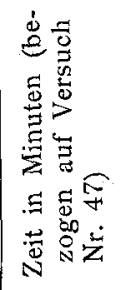 } & \multicolumn{2}{|c|}{$\begin{array}{l}\text { Gaszusammen- } \\
\text { setzung }\end{array}$} & \multicolumn{2}{|c|}{$\begin{array}{l}\text { Durchschlags- } \\
\text { spannung }\end{array}$} \\
\hline & & & & $\begin{array}{l}\text { Volum- } \\
\text { prozent } \\
\mathrm{N}_{2} \mathrm{O}_{5}\end{array}$ & $\begin{array}{l}\text { Volum- } \\
\text { prozent } \\
\mathrm{O}_{3}\end{array}$ & \multirow{2}{*}{$\begin{array}{c}E_{a} \\
\text { Gesamt- } \\
\text { span- } \\
\text { nung }\end{array}$} & \multirow{2}{*}{$\begin{array}{c}E_{i} \\
\text { Span- } \\
\text { nung an } \\
\text { der Gas- } \\
\text { strecke }\end{array}$} \\
\hline & & & & \multicolumn{2}{|c|}{$\begin{array}{c}\text { bezogen auf. } \\
\text { das Anfangsgas }\end{array}$} & & \\
\hline $29(22)$ & $746 \cdot 5$ & $15 \cdot 5$ & 0 & 0 & 0 & 7600 & 6450 \\
\hline 45 & $740 \cdot 1$ & $14 \cdot 0$ & 4 & $0 \cdot 17$ & $2 \cdot 35$ & 9800 & 8300 \\
\hline 44 & $738 \cdot 9$ & $14 \cdot 9$ & 5 & $0 \cdot 19$ & $2 \cdot 53$ & 10000 & 8500 \\
\hline 43 & $742 \cdot 0$ & $14 \cdot 6$ & 10 & $0 \cdot 28$ & $2 \cdot 48$ & 10400 & 8850 \\
\hline 48 & $745 \cdot 0$ & $12 \cdot 4$ & 30 & $0 \cdot 45$ & $(3: 6)$ & 11000 & 9350 \\
\hline 42 & $739 \cdot 0$ & $15 \cdot 5$ & 60 & $0 \cdot 8$ & $(3 \cdot 3)$ & 11200 & 9500 \\
\hline $47(40)$ & $735 \cdot 1$ & $15 \cdot 8$ & $158(145)$ & $1 \cdot 8$ & 0 & 11800 & 10000 \\
\hline 40 & - & - & $(150)$ & - & 0 & 10200 & 8650 \\
\hline 40 & - & - & (151) & - & 0 & 9900 & 8400 \\
\hline 41 & - & - & (156) & - & 0 & 9700 & 8250 \\
\hline 51 & $748 \cdot 1$ & $11 \cdot 1$ & - & \multicolumn{2}{|c|}{ Endzustand } & 8050 & 6850 \\
\hline 33 & $739 \cdot 2$ & $17 \cdot 1$ & 一 & \multicolumn{2}{|c|}{ Endzustand } & 7800 & 6600 \\
\hline
\end{tabular}

In Fig. 6 sind die gewonnenen Werte graphisch dargestellt. Diagramm $A$ zeigt die Minderdruckkurve in Millimetern $\mathrm{Hg}$, sowie den Anstieg der $\mathrm{N}_{2} \mathrm{O}_{5}$-Konzentration, ausgedrückt in Volumprozenten NO. Diagramm $B$ verzeichnet den steilen Anstieg der Spannung an der Gasstrecke bei wachsender $\mathrm{N}_{2} \mathrm{O}_{5}$ - und $\mathrm{O}_{3}$-Konzentration, Diagramm $C$ den gleichzeitigen Abfall der Stromstärke und Leistung.

Scharfe Veränderungen treten im Augenblick des $\mathrm{N}_{2} \mathrm{O}_{5}$ Zerfalles ein; die Spannung sinkt rasch, Strom und Leistung steigen wieder an; im stationären Endzustand sind die Werte von den bei reiner Luft gewonnenen wenig verschieden; vgl. Fig. 7.

Die Änderung aller elektrischen Größen wird im wesentlichen durch die Tatsache bedingt, daß die Durchschlags- 
spannung mit wachsender $\mathrm{N}_{2} \mathrm{O}_{5}$, beziehungsweise Ozonkonzentration stark anwächst (Diagramm $B$ ).
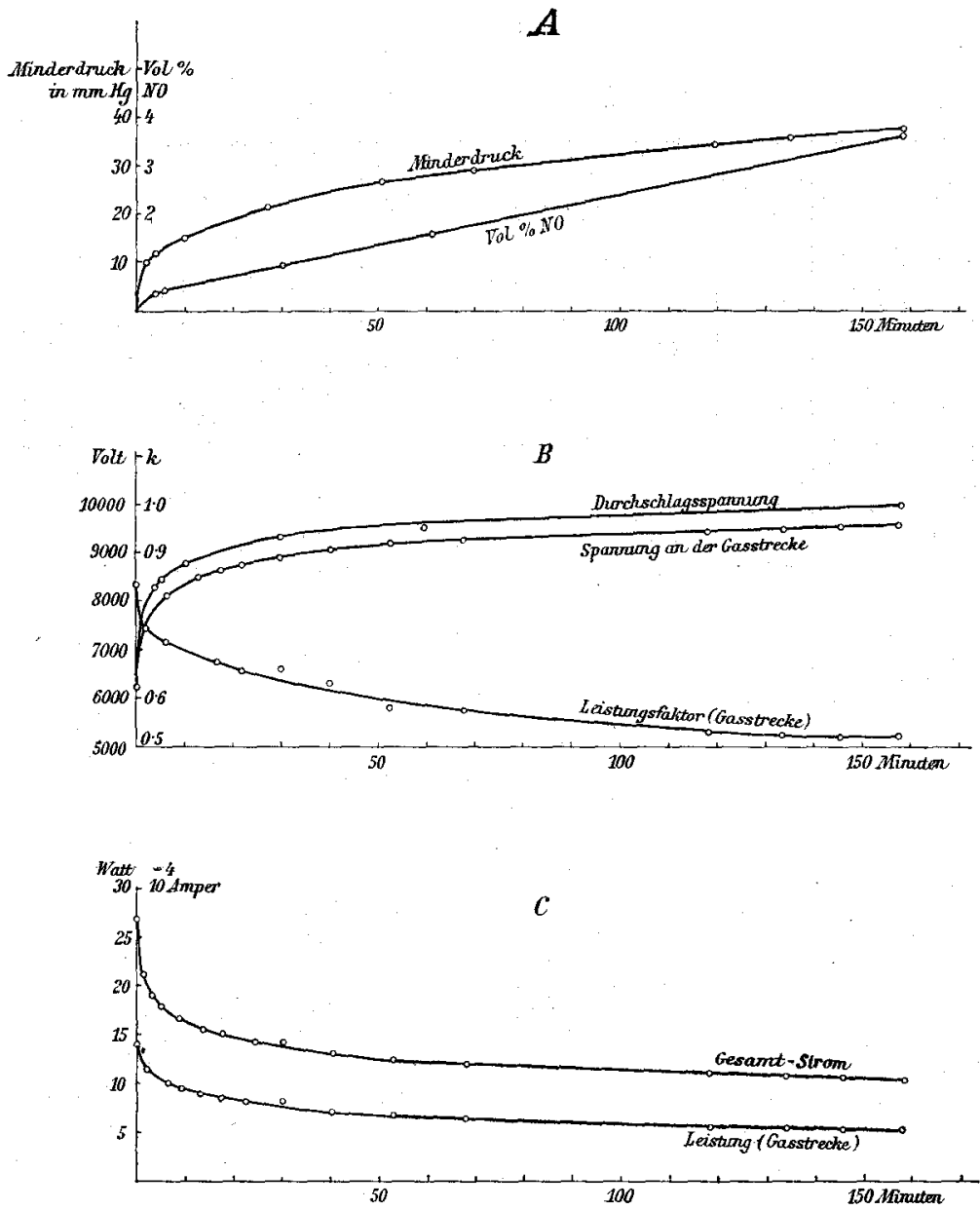

Fig. 6.

Wir fügen zur Vervollständigung des Bildes zwei Versuche an, die bei höheren Spannungen ausgeführt wurden (Nr. 51 und 39 [Fig. 7]).

Diese Versuche bieten außerdem ein Gesamtbild bis zum Eintreten des stationären Endzustandes. Der Anstieg der Stickoxydkonzentration ist hier als geradlinig angenommen. 


\section{Tabelle 8.}

\section{Versuch 51.}

$E=15100$ Volt $; E_{a}=15000$ Volt.

Luft, Gasdruck $748 \cdot 4 m m \mathrm{Hg}$.

\begin{tabular}{|c|c|c|c|c|c|c|c|c|c|c|}
\hline \multirow{2}{*}{ 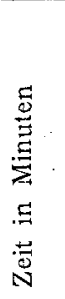 } & \multirow{2}{*}{ 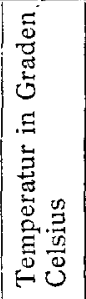 } & \multirow{2}{*}{ 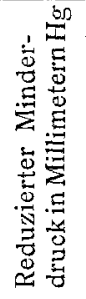 } & \multicolumn{2}{|c|}{$\begin{array}{l}\text { Volum- } \\
\text { prozent NO, } \\
\text { bezogen } \\
\text { auf das }\end{array}$} & \multirow{2}{*}{$J$} & \multirow{2}{*}{$L_{a}$} & \multirow{2}{*}{$k_{a}$} & \multirow{2}{*}{$E_{J}$} & \multirow{2}{*}{$L_{J}$} & \multirow{2}{*}{$k_{i}$} \\
\hline & & & 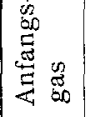 & 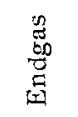 & & & & & & \\
\hline 0 & $11 \cdot 3$ & 0 & 0 & 0 & $(40 \cdot 5)$ & $(25 \cdot 3)$ & $0 \cdot 415$ & 6060 & $23 \cdot 1$ & 0.94 \\
\hline 1 & - & - & - & - & $(34)$ & $22 \cdot 5$ & $0 \cdot 44$ & 7200 & $20 \cdot 7$ & $0 \cdot 845$ \\
\hline $1 \cdot 5$ & - & - & - & - & $(33)$ & $22 \cdot 3$ & 0.45 & 7470 & $20 \cdot 6$ & 0.835 \\
\hline 2 & - & - & - & - & $32 \cdot 7$ & - & 一 & - & - & -- \\
\hline 3 & $11 \cdot 8$ & $13 \cdot 4$ & 一 & - & - & - & - & - & - & - \\
\hline 4 & - & - & - & - & $32 \cdot 0$ & - & - & - & - & - \\
\hline 5 & - & - & - & - & $(31 \cdot 7)$ & $22 \cdot 3$ & $0 \cdot 47$ & 7860 & $20 \cdot 6$ & $0 \cdot 83$ \\
\hline 6 & $12 \cdot 0$ & $15 \cdot 6$ & - & - & - & - & - & - & - & - \\
\hline 10 & - & - & - & - & $(30.9)$ & $22 \cdot 3$ & 0.48 & 8020 & $20 \cdot 6$ & 0.83 \\
\hline 11 & - & - & - & - & $30 \cdot 7$ & - & - & - & - & - \\
\hline 12 & $11 \cdot 8$ & $21 \cdot 6$ & - & - & - & - & - & - & - & - \\
\hline 16 & - & $\cdots$ & - & 一 & $30 \cdot 4$ & - & - & - & - & - \\
\hline $17 \cdot 5$ & - & - & - & - & $(29 \cdot 8)$ & $22 \cdot 3$ & $0 \cdot 495$ & 8300 & $20 \cdot 7$ & 0.83 \\
\hline $19 \cdot 5$ & $11 \cdot 9$ & $22 \cdot 3$ & - & - & - & - & - & - & - & - \\
\hline $26 \cdot 5$ & - & - & - & - & $29 \cdot 7$ & - & - & - & - & 一 \\
\hline $27 \cdot 5$ & $12 \cdot 0$ & $23 \cdot 9$ & - & - & $(29 \cdot 7)$ & $22 \cdot 2$ & 0.50 & 8400 & $20 \cdot 7$ & 0.83 \\
\hline 34 & - & $(25)$ & $2 \cdot 63$ & $2 \cdot 69$ & $30 \cdot 1$ & - & - & - & - & - \\
\hline $34 \cdot 5$ & $12 \cdot 2$ & $12 \cdot 3$ & - & - & $34 \cdot 6$ & - & - & 一 & -1 & - \\
\hline 35 & - & - & - & - & $(36)$ & $22 \cdot 6$ & 0.42 & 6700 & $20 \cdot 6$ & $0 \cdot 855$ \\
\hline 36 & - & - & - & - & $38 \cdot 8$ & - & - & - & - & - \\
\hline $36 \cdot 5$ & $12 \cdot 2$ & $2 \cdot 7$ & - & - & - & - & - & $=$ & - & 一 \\
\hline $37 \cdot 5$ & - & - & - & - & $40 \cdot 4$ & - & - & - & - & - \\
\hline 39 & - & - & - & 一 & $(41 \cdot 2)$ & $23 \cdot 3$ & 0.36 & 5350 & $21 \cdot 0$ & $0 \cdot 95$ \\
\hline 40 & $12 \cdot 4$ & $1 \cdot 1$ & $\cdots$ & - & - & - & - & - & - & - \\
\hline 47 & $12 \cdot 1$ & $0 \cdot 3$ & - & - & - & - & - & - & - & - \\
\hline 51 & - & - & - & - & $(43 \cdot 8)$ & $23 \cdot 2$ & $0 \cdot 35$ & 4900 & 20.6 & $0 \cdot 96$ \\
\hline 52 & $11 \cdot 7$ & $0 \cdot 3$ & - & - & $43 \cdot 8$ & - & - & - & - & - \\
\hline
\end{tabular}


Versuch Nr. 36 derselben Gruppe, der zu Anfang der Versuchsreihe aufgenommen wurde, zeigt vollkommen analogen Verlauf mit dem Obigen.

Tabelle 9.

Versuch 39.

$E=16850$ Volt $; E_{a}=16400$ Volt.

Luft, Gasdruck $730 \cdot 3 \mathrm{~mm} \mathrm{Hg}$.

\begin{tabular}{|c|c|c|c|c|c|c|c|c|c|c|}
\hline \multirow{2}{*}{ 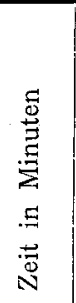 } & \multirow{2}{*}{ 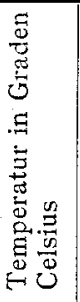 } & \multirow{2}{*}{ 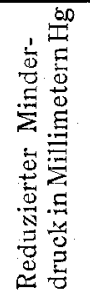 } & \multicolumn{2}{|c|}{$\begin{array}{l}\text { Volum- } \\
\text { prozent NO, } \\
\text { bezogen } \\
\text { auf das }\end{array}$} & \multirow{2}{*}{$J$} & \multirow{2}{*}{$L_{a}$} & \multirow{2}{*}{$k_{a}$} & \multirow{2}{*}{$E_{J}$} & \multirow{2}{*}{$L_{J}$} & \multirow{2}{*}{$k_{i}$} \\
\hline & & & 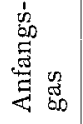 & 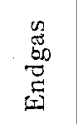 & & & & & & \\
\hline 0 & $15 \cdot 6$ & 0 & 0 & 0 & $(46)$ & $(31)$ & $0 \cdot 40$ & 6200 & $28 \cdot 0 \mid 0$ & 0.96 \\
\hline 0.5 & - & - & - & - & $43 \cdot 4$ & - & - & - & - & - \\
\hline 1 & - & - & - & - & $42 \cdot 3$ & - & - & - & - & - \\
\hline 2 & - & - & -1 & - & $(41 \cdot 5)$ & $30 \cdot 5$ & $0 \cdot 45$ & 7400 & $28 \cdot 1$ & 0.915 \\
\hline 3 & $15 \cdot 8$ & $12 \cdot 5$ & - & - & $40 \cdot 3$ & - & - & - & - & - \\
\hline 5 & $16 \cdot 0$ & $13 \cdot 8$ & - & - & $39 \cdot 7$ & - & - & - & - & - \\
\hline 6 & - & - & -1 & - & $39 \cdot 4$ & - & - & - & - & - \\
\hline 7 & - & - & -1 & - & $(38)$ & $29 \cdot 8$ & $0 \cdot 465$ & 7870 & $27 \cdot 6 \mid 0$ & $\cdot 90$ \\
\hline 8 & - & - & -1 & - & $38 \cdot 4$ & - & - & - & - & - \\
\hline 10 & $16 \cdot 2$ & $18 \cdot 5$ & - & - & - & - & - & - & - & - \\
\hline $10 \cdot 5$ & - & $\ldots$ & - & - & $38 \cdot 4$ & - & - & - & - & - \\
\hline 12 & - & - & - & - & $(38 \cdot 2)$ & $30 \cdot 0$ & 0.48 & 8200 & $27 \cdot 8 \mid$ & 0.89 \\
\hline $14 \cdot 5$ & - & - & - & - & $38 \cdot 1$ & - & - & - & - & - \\
\hline 15 & $16 \cdot 5$ & $22 \cdot 3$ & -1 & - & - & - & - & - & - & - \\
\hline 21 & $15 \cdot 7$ & $26 \cdot 6$ & - & - & $38 \cdot 2$ & - & - & - & - & - \\
\hline 23 & - & $(28)$ & $2 \cdot 95$ & 3.06 & $(38 \cdot 2)$ & $(29 \cdot 9)$ & $0 \cdot 48$ & 8200 & $27 \cdot 8$ & 0.89 \\
\hline 24 & - & - & - & - & $45 \cdot 1$ & - & - & - & - & - \\
\hline $24 \cdot 5$ & - & - & - & - & $46 \cdot 1$ & - & $\dot{-}$ & - & - & - \\
\hline 29 & - & - & - & - & $49 \cdot 0$ & - & - & - & - & - \\
\hline 30 & $15 \cdot 2$ & 0.3 & - & - & $(49 \cdot 0)$ & $30 \cdot 2$ & 0.375 & 5700 & $26 \cdot 9$ & 0.965 \\
\hline
\end{tabular}

Chemie-Heft Nr. 5. 
Versuch 51.
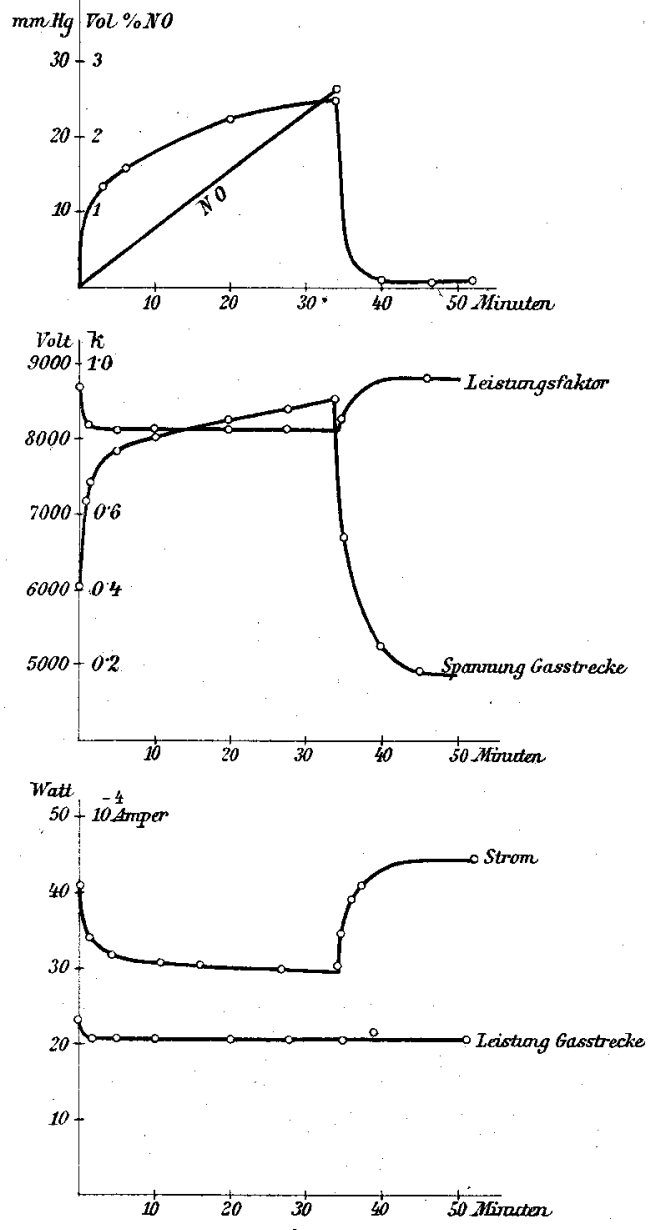

Versuch 39.
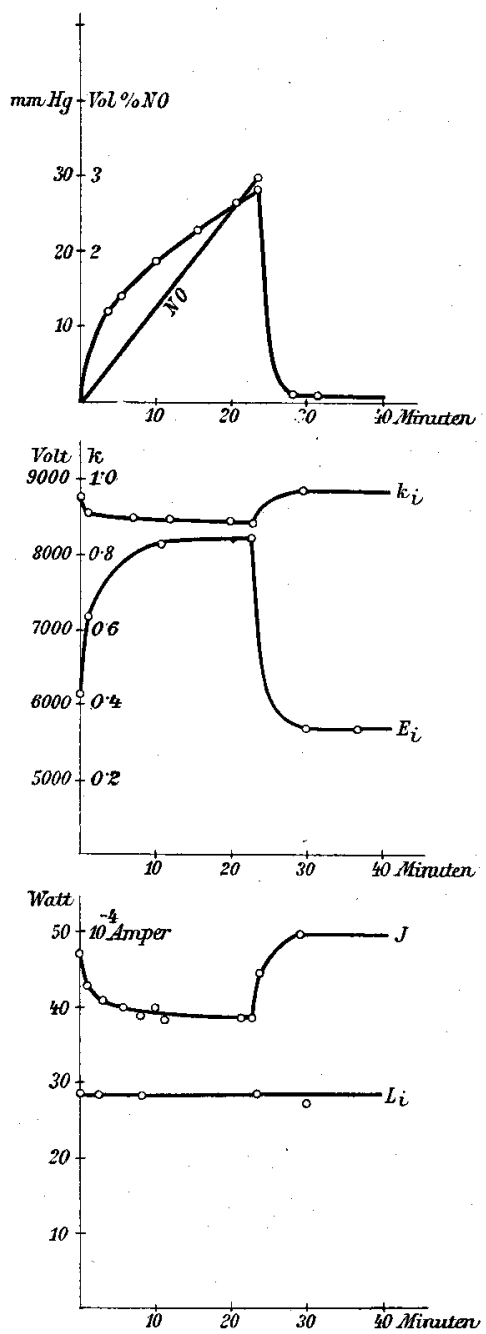

Fig. 7. 
Im Anschluß hieran erwähnen wir noch zwei im gleichen Siemensrohr, aber unter vermindertem Gasdruck (1/2 Atmosphäre) ausgeführte Gesamtversuche:

Tabelle 10.

Versuch 50.

$$
E_{\alpha}=10000 \text { Volt. }
$$

Luft, Gasdruck $370.1 \mathrm{~mm} \mathrm{Hg}$.

\begin{tabular}{|c|c|c|c|c|c|c|c|c|c|c|}
\hline 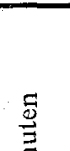 & 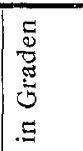 & 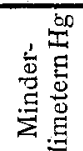 & $\begin{array}{r}\text { Vol } \\
\text { prozen } \\
\text { bezc } \\
\text { auf }\end{array}$ & $\begin{array}{l}\text { um- } \\
\text { it No, } \\
\text { gen } \\
\text { das }\end{array}$ & & & & & & \\
\hline 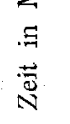 & 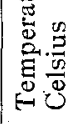 & 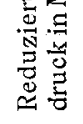 & 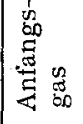 & 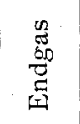 & & & & & & \\
\hline 0 & $11 \cdot 2$ & 0 & 0 & 0 & $(29)$ & $(10 \cdot 4)$ & 0.36 & 3400 & $8 \cdot 9$ & 0.90 \\
\hline 0.5 & - & - & - & - & $27 \cdot 6$ & - & - & - & - & - \\
\hline 1 & - & - & - & - & $26 \cdot 8$ & - & - & - & - & - \\
\hline 2 & - & - & - & - & $(26 \cdot 8)$ & $10 \cdot 3$ & 0.38 & 3800 & $8 \cdot 9$ & 0.875 \\
\hline 3 & $11 \cdot 5$ & $13 \cdot 1$ & - & - & - & - & - & - & - & - \\
\hline 4 & - & - & - & - & $(26 \cdot 8)$ & $10 \cdot 2$ & 0.38 & 3800 & 8.8 & 0.865 \\
\hline 6 & $11 \cdot 8$ & $16 \cdot 2$ & - & - & $\therefore$ & - & $\because$ & - & - & - \\
\hline $7 \cdot 5$ & - & - & - & - & $26 \cdot 9$ & - & - & - & -1 & - \\
\hline 9 & - & - & - & - & $(26 \cdot 7)$ & $10 \cdot 2$ & 0.38 & 3830 & 8.8 & $0 \cdot 86$ \\
\hline 10 & $12 \cdot 1$ & $20 \cdot 4$ & - & - & - & - & - & - & - & - \\
\hline 12 & - & - & - & - & $26 \cdot 5$ & $10 \cdot 2$ & $0 \cdot 385$ & 3900 & $8 \cdot 8$ & 0.85 \\
\hline 13 & - & - & - & - & $26 \cdot 4$ & - & - & - & - & - \\
\hline 15 & $12 \cdot 0$ & $23 \cdot 6$ & - & - & $26 \cdot 2$ & - & - & - & 一 & - \\
\hline 17 & - & $(25)$ & $2 \cdot 63$ & $2 \cdot 68$ & $(26 \cdot 5)$ & $9 \cdot 9$ & 0.38 & 3850 & $8 \cdot 5$ & 0.835 \\
\hline $19 \cdot 5$ & $11 \cdot 9$ & 0.6 & - & - & - & - & - & - & - & - \\
\hline $20 \cdot 5$ & - & - & - & - & $(28 \cdot 2)$ & $9 \cdot 5$ & 0.34 & 3350 & 8.0 & $0 \cdot 85$ \\
\hline 22 & $11 \cdot 9$ & 0.2 & - & - & $29 \cdot 1$ & $9 \cdot 4$ & $0 \cdot 325$ & 3150 & $7 \cdot 9$ & 0.86 \\
\hline
\end{tabular}


Tabelle 11.

Versuch 49.

$$
\begin{gathered}
E=12300 \text { Volt } ; E_{a}=12250 \text { Volt. } \\
\text { Luft, Gasdruck }=370 \mathrm{~mm} \mathrm{Hg.}
\end{gathered}
$$

\begin{tabular}{|c|c|c|c|c|c|c|c|c|c|c|}
\hline \multirow{2}{*}{ 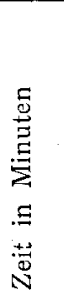 } & \multirow{2}{*}{ 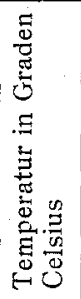 } & \multirow{2}{*}{ 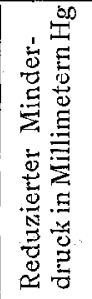 } & \multicolumn{2}{|c|}{$\begin{array}{c}\text { Volum- } \\
\text { prozent NO, } \\
\text { bezogen } \\
\text { auf das }\end{array}$} & \multirow{2}{*}{$J$} & \multirow{2}{*}{$L_{a}$} & \multirow{2}{*}{$k_{a}$} & \multirow{2}{*}{$E_{J}$} & \multirow{2}{*}{$L_{J}$} & \multirow{2}{*}{$\hbar_{i}$} \\
\hline & & & 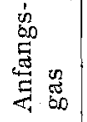 & 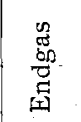 & & & & & & \\
\hline 0 & $11 \cdot 0$ & 0 & 0 & 0 & $(36 \cdot 0)$ & $(13 \cdot 0)$ & $0 \cdot 295$ & 3450 & $11 \cdot 1$ & 0.895 \\
\hline 0.5 & $11 \cdot 4$ & $12 \cdot 3$ & - & - & $34 \cdot 3$ & 一 & - & - & - & - \\
\hline 1 & - & - & - & - & $(34)$ & $13 \cdot 5$ & $0 \cdot 32$ & 3950 & $11 \cdot 8$ & $0 \cdot 88$ \\
\hline $1 \cdot 5$ & - & - & - & - & $33 \cdot 8$ & $13 \cdot 8$ & $0 \cdot 33$ & 4100 & $12 \cdot 0$ & 0.865 \\
\hline 3 & - & - & - & - & $33 \cdot 4$ & - & 一 & - & - & - \\
\hline 5 & $11 \cdot 8$ & $15 \cdot 6$ & - & - & $33 \cdot 3$ & - & - & - & - & - \\
\hline 7 & - & - & - & - & $(33 \cdot 2)$ & $14 \cdot 4$ & 0.35 & 4100 & $11 \cdot 7$ & $0 \cdot 85$ \\
\hline 9 & - & $(18 \cdot 5)$ & $1 \cdot 95$ & $1 \cdot 97$ & $(33 \cdot 5)$ & $13 \cdot 5$ & $0 \cdot 33$ & 4100 & $11 \cdot 7$ & 0.85 \\
\hline 10 & 12.1 & $11 \cdot 9$ & - & - & $33 \cdot 7$ & - & - & - & - & - \\
\hline 11 & - & - & - & - & $34 \cdot 5$ & - & - & - & - & - \\
\hline 12 & - & - & - & - & $(35)$ & $12 \cdot 2$ & $0 \cdot 28$ & 3500 & $10 \cdot 3$ & $0 \cdot 84$ \\
\hline $12 \cdot 5$ & $12 \cdot 1$ & 0 & - & - & - & - & - & - & - & - \\
\hline 14 & - & - & - & - & $(36 \cdot 1)$ & $12 \cdot 0$ & 0.27 & 3250 & $10 \cdot 0$ & $0 \cdot 86$ \\
\hline 15 & $12 \cdot 1$ & $0 \cdot 6$ & - & - & $36 \cdot 1$ & - & - & - & - & - \\
\hline 19 & - & - & - & - & - & - & - & - & - & - \\
\hline
\end{tabular}

Der Unterschied in den Versuchsbedingungen bei 50 und 49 gegenüber den vorher mitgeteilten Versuchen ist durch die niedrigere Durchschlagsspannung an der Gasstrecke gekennzeichnet. Hieraus ergibt sich für gleiche Stromdichte niedrigere Spannung an der Gasstrecke und geringerer Energieverbrauch sowie entsprechend geänderte Leistungsfaktoren. Die Verhältnisse sind auf Grund der charakteristischen Kurven (Fig. 5; p. 332) vorauszusehen. 
Trotz veränderter Bedingungen ist aber der Verlauf der elektrischen Größen analog dem bei den Versuchen unter höherem Gasdruck, wie ja auch die chemischen Vorgänge qualitativ die gleichen sind.

Die Durchschlagsspannung, damit die Spannung an der Gasstrecke und alle anderen elektrischen Größen werden durch die chemische Zusammensetzung des Gases äußerst empfindlich beeinflußt. Der Zerfall der schweren Moleküle nach dem Druckminimum führt $\mathrm{zu}$ einem raschen Sinken der stark gestiegenen Durchschlagsspannung; parallel damit fällt die Spannung an der Gasstrecke, während die Stromstärke wieder steigt. Im chemisch-stationären Endzustand bleiben auch die elektrischen Größen konstant. Hier ist beachtenswert, daß trotz etwas höherer Durchschlagsspannung gegenüber reiner Luft die Spannung an der Gasstrecke merklich niedriger, die Stromstärke größer ist als im Anfangszustand.

Die starke Beeinflussung der Durchschlagsspannung durch die chemische Zusammensetzung des Gases hängt innig mit den Veränderungen der mittleren freien Weglängen zusammen; denn die Entladung setzt erst dann ein, wenn die Spannungsverteilung im elektrischen Felde an einem Punkte den positiven Ionen über die freie Weglänge die Ionisierungsenergie verleiht.

Die in vorliegender Arbeit ausführlicher mitgeteilten Versuche wurden in demselben Siemensrohr bei Veränderung der Stromdichte und der Durchschlagsspannung (Gasdruck) ausgeführt. Hieran schlossen sich Versuchsreihen in anderen Ozonisatoren (geänderte Elektrodendistanz, geänderter Innenradius), sowie unter Verwendung anderer Stromformen (kondensierter Wechselstrom, unterbrochener Gleichstrom). Hierbei zeigte sich stets in analoger Weise der enge Zusammenhang zwischen dem chemischen Verlauf der Stickstoffoxydation und der Veränderung der elektrischen Größen an der Gasstrecke der Siemensröhre.

\section{Zusammenfassung.}

1. Zwecks Messung der elektrischen Größen der Siemensröhre wurde die Methodik der Anwendung des Binantelektro- 
meters nach Dolezalek ausgebildet, insbesondere für die Bestimmung der Leistung bei großer Phasenverschiebung zwischen Strom und Spannung. Die durch direkte Messung ermittelten elektrischen Größen an den äußeren Belägen der Siemensröhre ermöglichen die Berechnung der elektrischen Größen an der Gasstrecke.

2. Die Veränderungen der elektrischen Größen wurden im Zusammenhange mit den chemischen Vorgängen bei der Stickstoffoxydation in Gegenwart von Ozon verfolgt.

3. Es besteht eine scharfe Abhängigkeit der Durchschlagsspannung von der sich stetig ändernden chemischen $\mathrm{Zu}$ sammensetzung des Gases; Ozon und Stickstoffpentoxyd erhöhen schon in geringen Konzentrationen die Durchschlagsspannung des. Stickstoff-Sauerstoffgemisches um ein Beträchtliches. Wesentlich diese Tatsache bedingt, daß die elektrischen Größen, vor allem Stromstärke und Spannung an der Gasstrecke, durch die im Laufe der Entladungen auftretenden chemischen Veränderungen auf das empfindlichste beeinflußt werden.

Bei der Durchführung dieser Versuche hatten wir uns in besonders dankenswerter Weise der Unterstützung der Bosnischen. Elektrizitäts-Aktiengesellschaft in Wien $\mathrm{zu}$ erfreuen. 